\title{
BRANCHED COVERINGS. I
}

\author{
BY
}

R. E. STONG ${ }^{1}$

\begin{abstract}
This paper analyzes the possible cobordism classes $[M]-(\operatorname{deg} \phi)[N]$ for $\phi: M \rightarrow N$ a smooth branched covering of closed smooth manifolds. It is assumed that the branch set is a codimension 2 submanifold. The results are a fairly complete description in the unoriented case, a partial description in the oriented case, and a detailed analysis of the case in which $N$ is a sphere.
\end{abstract}

1. Introduction. The purpose of this note is to describe the possible cobordism classes $[M]-(\operatorname{deg} \phi)[N]$ where $\phi: M \rightarrow N$ is a smooth branched covering of closed smooth manifolds.

It is well known that for a genuine covering $\phi: M \rightarrow N$ one has $[M]=(\operatorname{deg} \phi)[N]$ in unoriented cobordism or in oriented cobordism if $M$ and $N$ are oriented manifolds. Thus, the class $[M]-(\operatorname{deg} \phi)[N]$ depends entirely upon the branching behavior. For this definition, the choice here is to follow Berstein and Edmonds [2] including a smoothness hypothesis or more specifically Brand [3] since the differentiable structures will be assumed to satisfy his regularity condition. Briefly then,

Definition. A branched covering is a smooth map $\phi: M^{n} \rightarrow N^{n}$ between smooth manifolds which is finite-to-one and an open map. The singular set $\Sigma_{\phi}$ is the set of points of $M$ at which $\phi$ is not a local homeomorphism, and the branch set $B_{\phi}$ is the image under $\phi$ of the singular set. Assume that the branch set is a smooth codimension 2 submanifold of $N$.

According to [2], the map $\phi: \phi^{-1} B_{\phi} \rightarrow B_{\phi}$ is then an ordinary covering and looks like a union of maps $\cup_{j} B_{i j} \rightarrow B_{i}$, where $B_{i}$ is a component of $B_{\phi}$ and each $B_{i j} \rightarrow B_{i}$ is a covering of degree $r_{i j}$. If $\nu_{i j}$ is the normal bundle of $B_{i j}$ in $M$ and $\nu_{i}$ the normal bundle of $B_{i}$ in $N$, then $\phi^{*} \nu_{i} \mid B_{i j}$ looks like a quotient of $\nu_{i j}$ by an identification of degree $d_{i j}$ (the local branching degree) on the fibers; i.e. locally $\phi$ is the map

$$
R^{n-2} \times \mathbf{C} \rightarrow R^{n-2} \times \mathbf{C}:(x, z) \rightarrow\left(x, z^{d_{i j}}\right) .
$$

Of course, the local degrees add up, so that

$$
\text { degree } \phi=\sum_{j} r_{i j} d_{i j}
$$

(which is constant on each component of $N$ ). Up to cobordism, the specific differential structure on $M$ is irrelevant, and so additionally one assumes Brand's conditions hold.

Received by the editors December 1, 1981 and, in revised form, March 23, 1982.

1980 Mathematics Subject Classification. Primary 57R75, 55R55.

' I am indebted to Gordon Keller and to my son, Richard Stong, for assistance, to the National Science Foundation for financial support, and particularly to Larry Smith, with whom I worked briefly on cobordism of branched covers. 
Note. For the orthogonal 2-plane bundle $\nu_{i j}$ over $B_{i j}$, one may form a quotient $\mu_{d_{i j}}\left(\nu_{i j}\right)$ by identifying vectors in fibers which differ by an angle which is an integral multiple of $2 \pi / d_{i j}$. This is again a 2-plane bundle and is identified with $\phi^{*} \nu_{i} \mid B_{i j}$. If one observes that two 2-plane bundles over a space having the same first StiefelWhitney class have a tensor product, $\mu_{d_{i j}}\left(\nu_{i j}\right)$ is just the $d_{i j}$ th tensor power of $\nu_{i j}$.

From a cobordism standpoint, one should first observe that there is a cobordism group of branched coverings of degree $d$ for closed manifolds, and that the Conner-Floyd [6] methods can actually be used successfully to analyze branched coverings with a fixed degree almost as if one were working with a group action. Specifically, one has an exact sequence

$$
\begin{aligned}
\cdots & \rightarrow \Omega_{n}(d \text {-fold cover }) \stackrel{i_{*}}{\rightarrow} \Omega_{n}(d \text {-fold branched cover }) \\
& \stackrel{j_{*}}{\rightarrow} \Omega_{n}(d \text {-fold branched cover, unbranched } \partial) \stackrel{\partial}{\rightarrow} \Omega_{n-1}(d \text {-fold cover }) \stackrel{i_{*}}{\rightarrow} \ldots
\end{aligned}
$$

in either oriented or unoriented cobordism. Clearly, $\Omega_{n}$ ( $d$-fold cover) is just the usual bordism of $B \Sigma_{d}$ and by the work of Brand [4], $\Omega$ ( $d$-fold branched cover) is the usual bordism of Brand's classifying space $B_{d}$. The relative group is $\Omega_{n}\left(B_{d}, B \Sigma_{d}\right)$ and is the reduced bordism of a certain wedge of very nasty Thom spaces (i.e. $B_{d}$ is obtained from $B \Sigma_{d}$ by attaching a union of disc bundles of 2-plane bundles by means of maps of their sphere bundles into $B \Sigma_{d}$ ).

In the special case when $d=2$, a branched cover is nothing more than an involution with codimension two fixed point set, and, in fact, one completely understands the Conner and Floyd analysis of this case. One has

Proposition 1. Assigning to a 2-fold branched cover $\phi: M^{n} \rightarrow N^{n}$ the class of $N$ and the class of $\tilde{\nu}$ over $\phi^{-1} B_{\phi}$ restricted to the self-intersection of $\phi^{-1} B_{\phi}$ in $M$ defines isomorphisms

$$
\Omega_{n}(2 \text {-fold branched cover }) \cong \Omega_{n} \oplus \Omega_{n-4}\left(\mathrm{BO}_{2}\right)
$$

and

$$
\mathfrak{N}_{n}(2 \text {-fold branched cover }) \cong \mathfrak{N}_{n} \oplus \mathfrak{N}_{n-4}\left(\mathrm{BO}_{2}\right)
$$

In the unoriented case, the analysis is not overly difficult and one finds

Proposition 2. If $\phi: M^{n} \rightarrow N^{n}$ is a branched cover of closed manifolds, then $\left[M^{n}, \phi\right]-(\operatorname{deg} \phi)\left[N^{n}\right.$, identity $]$ in $\mathfrak{N}_{n}\left(N^{n}\right)$ is the class of the map

$$
\bigcup\left\{R P\left(\nu_{i j} \oplus 1\right) \mid d_{i j} \text { is even }\right\} \rightarrow B_{\phi} \subset N,
$$

i.e., one takes the union of the $D\left(\nu_{i j}\right) /\left\{x \sim-x \mid x \in S\left(\nu_{i j}\right)\right\}$ for $d_{i j}$ even, projects onto $B_{i j}$, composes with $\phi$ into $B_{i}$, and then includes in $N$.

Proposition 3. The set of classes $\left[M^{n}\right]-d\left[N^{n}\right]$ in $\mathfrak{N}_{n}$ for $\phi: M^{n} \rightarrow N^{n}$ a d-fold branched covering of closed $n$ dimensional manifolds is

$$
\left\{\alpha \in \mathfrak{N}_{n} \mid w_{1}^{n}(\alpha)=0\right\},
$$

if $d \geqslant 2, n>0$. 
In the oriented case, one has

Proposition 4. If $\alpha \in \Omega_{n}, n>0$, there is an odd integer $k$ and a branched covering $\phi: M^{n} \rightarrow N^{n}$ of closed oriented manifolds with

$$
\left[M^{n}\right]-(\operatorname{deg} \phi)\left[N^{n}\right]=k \alpha \text { in } \Omega_{*} .
$$

Note. There is such an odd integer $k$ for coverings of degree two. If one specifies the degree $d \geqslant 2$, the $k$ needed may vary.

In the above one cannot take $k=1$ in general. One has

Proposition 5. If $n=2 k(p-1)$ with $p$ an odd prime and $\alpha \in \Omega_{n}$ is the class $\left[M^{n}\right]-d\left[N^{n}\right]$ for some d-fold branched covering $\phi: M^{n} \rightarrow N^{n}$ of closed oriented manifolds with $d<2 p$, then one has

$$
s_{k(p-1) / 2}(\mathfrak{p})[\alpha] \equiv 0 \quad(\bmod p)
$$

where $s_{m}(\mathfrak{p})$ is the primitive characteristic class.

ReMARKS. In particular, for $n=2 k(p-1)$ and $n / 2+1$ not a power of $p$, the class $\alpha$ cannot be indecomposable in $\Omega_{*} / p \Omega_{*}$.

A number of results will be given characterizing the values $s_{m}(\mathfrak{p})[\alpha]$ for $\alpha \in \Omega_{4 m}$ realized by a $d$-fold branched covering.

In later work, appearing as part II of this paper, it is shown that $s_{\left(i_{1}((p-1) / 2), \ldots, i_{r}((p-1) / 2)\right)}(\mathfrak{p})[\alpha] \equiv 0 \bmod p$ for all $\left(i_{1}, \ldots, i_{r}\right)$ without the restriction $d<2 p$, and precise divisibility of the numbers $s_{m}(\mathfrak{p})[\alpha]$ is obtained.

Recently, Edmonds proved [7] that no simply connected closed Spin 4-manifold of nonzero signature can be a 2-fold branched covering of the 4-sphere. His argument can be extended, and one has

Proposition 6. If $\phi: M^{n} \rightarrow N^{n}$ is a branched covering of closed oriented manifolds with oriented branch set $B_{\phi}$ and $H^{2}(N ; Q)=0$, then $\left[M^{n}\right]-(\operatorname{deg} \phi)\left[N^{n}\right] \in$ Tor $\Omega$.

Proposition 7. If $\phi: M^{n} \rightarrow N^{n}$ is a branched covering of closed oriented manifolds with $H^{4}(N ; Q)=0$, then $\left[M^{n}\right]-(\operatorname{deg} \phi)\left[N^{n}\right] \in$ Tor $\Omega_{*}$.

Proposition 8. Let $\phi: M^{n} \rightarrow S^{n}$ be a branched covering with $M^{n}$ closed and if $n=4$ assume $B_{\phi}$ orientable. Then $M^{n}$ is orientable and $\left[M^{n}\right] \in$ Tor $\Omega_{*}$. If $M^{n}$ is a Spin manifold or if $B_{\phi}$ is orientable, then $\left[M^{n}\right]=0$ in $\Omega_{*}$.

By calculating Stiefel-Whitney numbers one then has

Proposition 9. If $\phi: M^{n} \rightarrow S^{n}$ is a branched covering then $M^{n}$ bounds (as oriented manifold) if $n$ is even and greater than 4 or if $n$ is odd and $n+1$ does not have exactly two ones in its dyadic expansion.

2. Involutions. First, one considers 2-fold branched covers $\phi: M^{n} \rightarrow N^{n}$ and defines an involution $t: M \rightarrow M$ by the condition that $\phi^{-1} \phi(m)=\{m, t m\}$. The fixed point set of $t$ is $\phi^{-1} B_{\phi} \cong B_{\phi}$. If in the oriented case, one insists that the orientation of $N$ on $N-B_{\phi}$ lift back to the orientation of $M-\phi^{-1} B_{\phi}$, and with that choice, $t$ is an orientation preserving involution. 
For the unoriented case, one has the Conner and Floyd [6, (28.1)] exact sequence

$$
0 \rightarrow \mathfrak{N}_{n}^{Z_{2}} \stackrel{F}{\rightarrow} \bigoplus_{k} \mathfrak{N}_{n-k}\left(B O_{k}\right) \stackrel{\partial}{\rightarrow} \mathfrak{N}_{n-1}\left(B Z_{2}\right) \rightarrow 0
$$

and restricting to codimension 2 fixed point set, a corresponding exact sequence

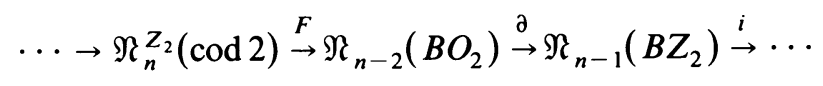

where $F$ takes the fixed point set with its normal bundle, $\partial$ assigns to $\xi^{2} \rightarrow F$ the antipodal involution on $S\left(\xi^{2}\right)$ or the double cover $S\left(\xi^{2}\right) \rightarrow R P\left(\xi^{2}\right)$, and $i$ assigns to a double covering the class of the free involution on the total space.

For an involution with codimension 2 fixed point set $\left(M^{n}, t\right)$, the quotient $M^{n} / t$ is again a manifold giving a homomorphism

$$
q: \mathfrak{R}_{n}^{Z_{2}}(\operatorname{cod} 2) \rightarrow \mathfrak{R}_{n}
$$

and the composite

$$
q i: \mathfrak{N}_{n}\left(B Z_{2}\right) \rightarrow \mathfrak{N}_{n}
$$

is the augmentation $\varepsilon\left[N \stackrel{f}{\rightarrow} B Z_{2}\right]=[N]$, and so image $\partial$ is contained in $\tilde{\mathfrak{R}}_{n}\left(B Z_{2}\right)=$ ker $\varepsilon$. Alternatively, $R P\left(\xi^{2}\right)$ is an $S^{1}$ bundle over $F$ and hence bounds.

From [6, (26.4)], one has a commutative diagram

$$
\begin{array}{ccc}
\mathfrak{N}_{n-2}\left(B O_{2}\right) & \stackrel{\partial}{\rightarrow} & \mathfrak{N}_{n-1}\left(B Z_{2}\right) \\
\uparrow I_{*} & & \downarrow \Delta \\
\mathfrak{N}_{n-2}\left(B O_{1}\right) & \stackrel{\partial}{\rightarrow} & \mathfrak{N}_{n-2}\left(B Z_{2}\right)
\end{array}
$$

where $I_{*}$ is the Whitney sum with a trivial line bundle, $\Delta$ is the Smith homomorphism, and the lower homomorphism $\partial$ is an isomorphism. Now $\Delta: \mathfrak{N}_{n-1}\left(B Z_{2}\right) \rightarrow$ $\mathfrak{R}_{n-2}\left(B Z_{2}\right)$ has kernel given by the classes of the trivial double covers (the class of $P\left[S^{0}, A\right]$ ), and maps $\tilde{\mathfrak{N}}_{n-1}\left(B Z_{2}\right)$ isomorphically to $\mathfrak{N}_{n-2}\left(B Z_{2}\right)$ (on bases $\left.\Delta\left(\left[S^{k}, A\right]-R P^{k}\left[S^{0}, A\right]\right)=\left[S^{k-1}, A\right]\right)$.

Thus the Conner-Floyd exact sequence becomes

$$
0 \rightarrow \mathfrak{N}_{n} \stackrel{i}{\rightarrow} \mathfrak{N}_{n}^{Z_{2}}(\operatorname{cod} 2) \stackrel{F}{\rightarrow} \mathfrak{N}_{n-2}\left(B O_{2}\right) \stackrel{\partial}{\rightarrow} \tilde{\mathfrak{N}}_{n-1}\left(B Z_{2}\right) \rightarrow 0
$$

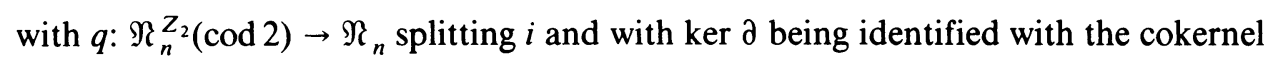
of

$$
I_{*}: \Re_{n-2}\left(B O_{1}\right) \rightarrow \Re_{n-2}\left(B O_{2}\right)
$$

One has the cofibration $B O_{1} \rightarrow B O_{2} \rightarrow M O_{2}$ from which coker $I_{*} \cong \tilde{\mathfrak{N}}_{n-2}\left(M O_{2}\right)$ $\cong \mathfrak{N}_{n-4}\left(B O_{2}\right)$, and recognizing the composite

$$
\mathfrak{N}_{n}^{Z_{2}}(\operatorname{cod} 2) \stackrel{F}{\rightarrow} \mathfrak{N}_{n-2}\left(B O_{2}\right) \rightarrow \tilde{\mathfrak{N}}_{n-2}\left(M O_{2}\right) \cong \mathfrak{N}_{n-4}\left(B O_{2}\right)
$$


as being the selfintersection of $F$ with the restriction of the normal bundle, one obtains

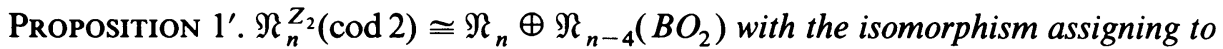
$\left(M^{n}, t\right)$ the class of $M^{n} / t$ and of $F^{n-2} \cap F^{n-2}$ with bundle $\left.\nu^{2}\right|_{F \cap F}$.

The oriented case is slightly more difficult with the analogue of the Conner-Floyd sequence being

$$
\cdots \rightarrow \Omega_{n}^{Z_{2}} \stackrel{F}{\rightarrow} \bigoplus_{k} \tilde{\Omega}_{n}\left(M O_{2 k}\right) \stackrel{\partial}{\rightarrow} \Omega_{n-1}\left(B Z_{2}\right) \stackrel{i_{*}}{\rightarrow} \cdots
$$

with the relative group of orientation preserving involutions which are free on the boundary being identified with $\bigoplus_{k} \tilde{\Omega}_{n}\left(M O_{2 k}\right)$ by assigning to $\left(V^{n}, t\right)$ the class of the map $V^{n} \rightarrow V_{k} M O_{2 k}$ sending a tubular neighborhood of $F^{n-2 k}$; i.e., $D\left(\nu^{2 k}\right)$, to $M O_{2 k}$ by $F^{n-2 k} \rightarrow B O_{2 k}$ and extending to the bundles $D\left(\nu^{2 k}\right) \rightarrow D\left(\gamma_{2 k}\right) \stackrel{c}{\rightarrow} M O_{2 k}$ where $c$ is the collapse, and sending the complement of these tubular neighborhoods to the common basepoint. (Orientation preserving involutions were first analyzed by Rosenzweig [15], but this description is due to Lee and Wasserman [12, p. 206].)

Restricting to a codimension 2 fixed point set gives

$$
\cdots \rightarrow \Omega_{n}^{Z_{2}}(\operatorname{cod} 2) \stackrel{F}{\rightarrow} \tilde{\Omega}_{n}\left(M O_{2}\right) \stackrel{\partial}{\rightarrow} \Omega_{n-1}\left(B Z_{2}\right) \stackrel{i_{*}}{\rightarrow} \cdots
$$

and as before one has $q: \Omega_{n}^{Z_{2}}(\operatorname{cod} 2) \rightarrow \Omega_{n}$ sending $\left(M^{n}, t\right)$ to the class of $M^{n} / t$, with $q i=\varepsilon: \Omega_{n}\left(B Z_{2}\right) \rightarrow \Omega_{n}$ so that image $\partial \subset \tilde{\Omega}_{n-1}\left(B Z_{2}\right) \cong \mathfrak{N}_{n-2}$.

Note. This isomorphism is due to Atiyah [1] and assigns to $f: P^{n-1} \rightarrow B Z_{2}$ with $\partial P$ mapping to the base point, with $\left(B Z_{2 *}\right)$ being thought of as $\left(M O_{1}, \infty\right)$ the submanifold $Q^{n-2} \subset P^{n-1}$ obtained by making the map transverse to $B O_{1} \subset M O_{1}$. $Q$ is an unoriented manifold and its normal line bundle in $P$ is just the orientation bundle.

Now consider the bundle $\pi^{*} \gamma_{2} \rightarrow D\left(\gamma_{2}\right)$ where $\gamma_{2}$ is the universal 2-plane bundle over $\mathrm{BO}_{2}$, for which one has a cofibration sequence

$$
\begin{aligned}
& T\left(\left.\pi^{*} \gamma_{2}\right|_{S\left(\gamma_{2}\right)}\right) \rightarrow T\left(\pi^{*} \gamma_{2}\right) \rightarrow D\left(\pi^{*} \gamma_{2}\right) / S\left(\pi^{*} \gamma_{2}\right) \cup D\left(\left.\pi^{*} \gamma_{2}\right|_{S\left(\gamma_{2}\right)}\right) \\
& \cup \\
& S\left(\gamma_{2}\right) \rightarrow D\left(\gamma_{2}\right)
\end{aligned}
$$

where $T$ denotes the Thom space. The projection $\pi: D\left(\gamma_{2}\right) \rightarrow B O_{2}$ is a homotopy equivalence and so $T\left(\pi^{*} \gamma_{2}\right) \cong M O_{2}$. The sphere bundle $S\left(\gamma_{2}\right)$ may be identified with $B O_{1}$ with the projection onto $B O_{2}$ pulling $\gamma_{2}$ back to $\gamma_{1} \oplus 1$, so that $T\left(\left.\pi^{*} \gamma_{2}\right|_{S\left(\gamma_{2}\right)}\right)$ may be identified with $T\left(\gamma_{1} \oplus 1\right) \cong \Sigma M O_{1}$ and so that the map $\Sigma M O_{1} \rightarrow M O_{2}$ is induced by $B O_{1} \rightarrow B O_{2}$ classifying the Whitney sum of $\gamma_{1}$ with a trivial line. Finally, the disc bundle $D\left(\pi^{*} \gamma_{2}\right)$ is the disc bundle of $\gamma_{2} \oplus \gamma_{2}$ over $B O_{2}$ and collapsing $S\left(\pi^{*} \gamma_{2}\right) \cup D\left(\left.\pi^{*} \gamma_{2}\right|_{S\left(\gamma_{2}\right)}\right) \cong S\left(\gamma_{2} \oplus \gamma_{2}\right)$ makes the cofiber just $M\left(\gamma_{2} \oplus \gamma_{2}\right)$. This one has a cofibration

$$
\Sigma M O_{1} \rightarrow M O_{2} \rightarrow M\left(\gamma_{2} \oplus \gamma_{2}\right)
$$


Applying the function $\tilde{\Omega}_{*}$, one has an exact sequence

$$
\cdots \rightarrow \tilde{\Omega}_{n}\left(\Sigma M O_{1}\right) \rightarrow \tilde{\Omega}_{n}\left(M O_{2}\right) \rightarrow \tilde{\Omega}_{n}\left(M\left(\gamma_{2} \oplus \gamma_{2}\right)\right) \rightarrow \tilde{\Omega}_{n-1}\left(\Sigma M O_{1}\right) \rightarrow \cdots
$$

and since $\gamma_{2} \oplus \gamma_{2}$ is an oriented vector bundle, one has a Thom isomorphism $\tilde{\Omega}_{n}\left(M\left(\gamma_{2} \oplus \gamma_{2}\right)\right) \cong \Omega_{n-4}\left(B O_{2}\right)$, while $\tilde{\Omega}_{n}\left(\Sigma M O_{1}\right) \cong \tilde{\Omega}_{n-1}\left(M O_{1}\right) \cong \Re_{n-2}$. One may easily check that the composite

$$
\mathfrak{N}_{n-2} \cong \tilde{\Omega}_{n}\left(\Sigma M O_{1}\right) \rightarrow \tilde{\Omega}_{n}\left(M O_{2}\right) \stackrel{\partial}{\rightarrow} \tilde{\Omega}_{n-1}\left(B Z_{2}\right) \cong \mathfrak{N}_{n-2}
$$

is the identity (one quick way to see this is to compare with the unoriented case with $\tilde{\Omega}_{n}\left(\Sigma M O_{1}\right) \rightarrow \tilde{\mathfrak{N}}_{n}\left(\Sigma M O_{1}\right)$ being the monomorphism $\Re_{n-2} \rightarrow \mathfrak{N}_{n-2}\left(B O_{1}\right)$ which takes the orientation cover. One has a commutative diagram

$$
\begin{aligned}
& \mathfrak{R}_{n-2} \quad \rightarrow \quad \tilde{\Omega}_{n}\left(M O_{2}\right) \quad \stackrel{\partial}{\rightarrow} \quad \mathfrak{R}_{n-2} \\
& \downarrow \text { mono } \quad \downarrow \quad \downarrow \text { mono } \\
& \mathfrak{N}_{n-2}\left(B O_{1}\right) \quad \rightarrow \quad \underset{\tilde{\mathfrak{N}}_{n}\left(M O_{2}\right)}{\rightarrow} \quad \rightarrow \quad \mathfrak{N}_{n-2}\left(B O_{1}\right) \\
& \mathfrak{N}_{n-2}\left(\mathrm{BO}_{2}\right)
\end{aligned}
$$

and the composite along the bottom is the identity.

One then has

$$
0 \rightarrow \Omega_{n} \stackrel{i_{*}}{\rightarrow} \Omega_{n}^{Z_{2}}(\operatorname{cod} 2) \stackrel{F}{\rightarrow} \tilde{\Omega}_{n}\left(M O_{2}\right) \stackrel{\partial}{\rightarrow} \Re_{n-2} \rightarrow 0
$$

with $q: \Omega_{n}^{Z_{2}}(\operatorname{cod} 2) \rightarrow \Omega_{n}$ splitting $i_{*}$ and with kernel $\partial$ being identified with $\Omega_{n-4}\left(\mathrm{BO}_{2}\right)$ via the exact sequence

$$
0 \rightarrow \mathfrak{N}_{n-2} \stackrel{j}{\rightarrow} \tilde{\Omega}_{n}\left(\mathrm{MO}_{2}\right) \rightarrow \Omega_{n-4}\left(\mathrm{BO}_{2}\right) \rightarrow 0
$$

with $j$ split by $\partial$.

This gives

Proposition $1^{\prime \prime}$. Assigning to $\left(M^{n}, t\right)$ the class of $M^{n} / t$ and $F^{n-2} \cap F^{n-2}$ with normal bundle $\nu^{2} \mid F \cap F$ gives an isomorphism

$$
\Omega_{n}^{Z_{2}}(\operatorname{cod} 2) \cong \Omega_{n} \oplus \Omega_{n-4}\left(B O_{2}\right) .
$$

One could modify this argument by using $\mathrm{BSO}_{2}$ rather than $\mathrm{BO}_{2}$ for involutions preserving orientation and with oriented codimension 2 fixed point set. It is, however, more reasonable to consider actions of $Z_{m}$, the cyclic group of order $m$ simultaneously with the orientation hypothesis being automatic except for $m=2$.

If one considers semifree $Z_{m}$ actions preserving orientation with codimension 2 fixed point set (assumed orientable if $m=2$, and in fact, oriented) then one has an exact sequence of Conner-Floyd type

$$
\cdots \stackrel{i}{\rightarrow} \Omega_{n}^{Z_{m}}(\text { semifree }) \stackrel{F}{\rightarrow} \underset{j}{\bigoplus} \Omega_{n-2}\left(B S O_{2}\right) \stackrel{\partial}{\rightarrow} \Omega_{n-1}\left(B Z_{m}\right) \stackrel{i}{\rightarrow} \cdots
$$

where the sum on $j$ is for $1 \leqslant j \leqslant(m-1) / 2$ and $(j, m)=1$. This indexing by $j$ corresponds to the classification of the nontrivial irreducible real representations, 
which are of the form multiplication by $\exp (2 \pi i j / m)$ on $\mathbf{C}$ with $1 \leqslant j \leqslant(m-1) / 2$, with $(j, m)=1$ giving the semifree representations. This choice of $j$ 's gives the normal bundle to $F^{n-2}$ a complex structure or orientation and hence orients $F$ (see Conner and Floyd [6, §38] for $m>2$, while for $m=2$ the orientation is chosen on $F)$.

One also has a cofibration for the $m$ th tensor power $\tilde{\gamma}_{2}^{m}$ of the bundle $\tilde{\gamma}_{2}$ over $\mathrm{BSO}_{2}$

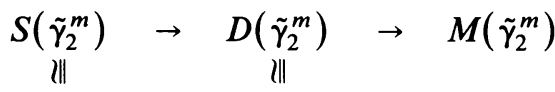

$$
\begin{aligned}
& \mathrm{BZ}_{m} \quad \mathrm{BSO}_{2}
\end{aligned}
$$

and applying $\Omega_{*}$, one obtains an exact sequence

$$
\begin{array}{cc}
\stackrel{i}{\rightarrow} \Omega_{n}^{Z_{m}}(\text { semifree, special }) \stackrel{F}{\rightarrow} \Omega_{n-2}\left(B S O_{2}\right) \stackrel{\partial}{\rightarrow} \Omega_{n-1}\left(B Z^{m}\right) \stackrel{i}{\rightarrow} \cdots \\
\Omega_{n}\left(B S O_{2}\right) & \tilde{\Omega}_{n}\left(M\left(\tilde{\gamma}_{2}^{m}\right)\right)
\end{array}
$$

where "special" means that $Z_{m}$ is to act by the standard representation in the (codim 2 assumed) normal bundle to the fixed set. This "special"-sequence maps into the above, and corresponding to a different choice of generator for $Z_{m}$ can be mapped in once for each $j$.

Note. Because image $\partial$ is finite, it follows that

$$
\theta: \bigoplus_{j} \Omega_{n}\left(B S O_{2}\right) \rightarrow \Omega_{n}^{Z_{m}}(\text { semifree })
$$

has image of finite index, or induces a rational isomorphism

$$
\theta: \frac{\oplus_{j} \Omega_{n}\left(B S O_{2}\right)}{\text { identify } \Omega_{n} \text { 's }} \rightarrow \Omega_{n}^{Z_{m}}(\text { semifree })
$$

by identifying the copies of $\Omega_{n} \cong \Omega_{n}$ (point) for the different $j$ 's. This says that some multiple of every semifree action is cobordant to a sum of actions, each having the same representation in the normal bundle to each component of $F$.

For $\Omega_{n}^{Z_{m}}$ (semifree, special $) \cong \Omega_{n}\left(B S O_{2}\right) \cong \Omega_{n}\left(D\left(\tilde{\gamma}_{2}^{m}\right)\right)$, the classifying space for the appropriate ramified coverings is $B S O_{2}=D\left(\tilde{\gamma}_{2}^{m}\right)$. The universal ramified covering is given by the infinite $m$-dric $\left\{z \in \mathbf{C P} P^{\infty} \mid \Sigma z_{i}^{m}=0\right\}$ ramified over $\mathbf{C P}=\mathrm{BSO}_{2}$ (see [16, §4, and particularly p. 308]). The standard basis over $\Omega_{*}$ of $\Omega_{*}\left(B S O_{2}\right)$ is given by the inclusions $\mathrm{CP}^{r} \rightarrow \mathrm{CP}=\mathrm{BSO}_{2}$ classifying the Hopf bundles and the induced $m$-fold ramified cover of the $m$-dric in $\mathbf{C} P^{r+1}, Q_{m}^{2 r}=\left\{z \in \mathbf{C} P^{r+1} \mid \Sigma_{0}^{r+1} z_{i}^{m}\right.$ $=0\}$, over $\mathbf{C} P^{r}$.

Note. These ramified coverings were studied by Hirzebruch [9] and Hattori [8]. Both incorrectly indicate that the $\mathrm{BSO}_{2}$ classifies semifree $Z_{m}$ actions, but one needs a single normal representation. The error is on line -2 , p. 260 of [9]; there is more than one way to include $G_{n}$ in $C^{*}$ corresponding to the different $j$ values. 
If one wishes to consider these semifree $Z_{m}$ actions as $m$-fold branched coverings with a single local branching degree $m$, i.e. $\cup_{j} B_{i j} \cong B_{i}$, and with $B_{\phi}$ oriented, one has a corresponding exact sequence

$$
\cdots \rightarrow \Omega_{n}(m \text {-fold, special }) \stackrel{F}{\rightarrow} \Omega_{n-2}\left(B S O_{2}\right) \stackrel{\partial}{\rightarrow} \Omega_{n-1}\left(B \Sigma_{m}\right) \stackrel{i}{\rightarrow} \cdots
$$

where "special" refers to the local degree only. The map $\partial$ factors through $\Omega_{n-1}\left(B Z_{m}\right)$, but one cannot distinguish a generator of $Z_{m}$ and hence has no dependence on the representation $j$. One has $\Omega_{n}(m$-fold special $) \cong \Omega_{n}\left(X_{m}\right)$ where $X_{m}$ is obtained by sewing $D\left(\tilde{\gamma}_{2}^{m}\right)$ to $B \Sigma_{m}$ along $S\left(\tilde{\gamma}^{m}\right) \cong B Z_{m}$, and is a special Brand classifying space.

CuRIosity. In the case $m=2$, the quadric $Q_{2}^{2 r} \subset \mathbf{C} P^{r+1}$ may be identified as the Grassmannian of oriented 2-planes in $R^{r+2}$ (see [11]). One may also observe that in the case $r$ even, $\left[Q_{2}^{2 r}\right]=2\left[H P^{r}\right]$ in $\Omega_{*}$, while for $r$ odd, both $Q_{2}^{2 r}$ and $\mathrm{C} P^{r}$ bound.

Note. It would appear that Brand's classifying space for 2-fold branched covers might be identifiable with $\mathrm{C} P^{\infty} /$ conjugation. Inside $\mathrm{CP}$ one has the quadric $\mathrm{BSO}_{2}$ and $R P^{\infty}$, with the normal bundle of $\mathrm{BSO}_{2}$ being $\tilde{\gamma}_{2}^{2}$, and with $\mathrm{CP} P^{\infty}$ being the union of tubular neighborhoods of these subsets. Conjugation fixes $\mathrm{RP}^{\infty}$ and acts on $\mathrm{BSO}_{2}$ as the standard free involution reversing orientation. Thus inside $\mathbf{C} P^{\infty} /$ conjugation one has copies of $B Z_{2}=R P^{\infty}$ and $\mathrm{BO}_{2}=B S O_{2} / Z_{2}$ with the complement of $R P^{\infty}$ being the disc bundle of a 2-plane bundle over $\mathrm{BO}_{2}$.

Comment. The ideas about $m$-fold covers above derive from my joint efforts with Larry Smith on cobordism of ramified covers.

3. Unoriented branchings. In order to describe the classes $\left[M^{n}\right]-(\operatorname{deg} \phi)\left[N^{n}\right]$ in cobordism, one must be able to compute the characteristic numbers, hence, needs to describe the characteristic classes. For this, one follows Brand [3].

Let $\phi: M^{n} \rightarrow N^{n}$ be a branched covering, and let $B=B_{\phi} \subset N$ be the branch set with normal bundle $\nu$. Let $\phi^{-1} B_{\phi}$ be written as the disjoint union of the submanifolds $\tilde{B}_{k}$, where $\tilde{B}_{k}$ is the set of points with local branching degree $k$, and let $\tilde{\nu}_{k}$ be the normal bundle of $\tilde{B}_{k}$ in $M$. If one chooses disjoint tubular neighborhoods $D\left(\tilde{\nu}_{k}\right)$ of the sets $\tilde{B}_{k}$, one may collapse the complement to obtain a map

$$
c: M^{n} \rightarrow \underset{k}{\bigvee} T\left(\tilde{\nu}_{k}\right)
$$

and by classification of $\tilde{\nu}_{k}$ one has $\tilde{B}_{k} \rightarrow B O_{2}$ covered by a map $T\left(\tilde{\nu}_{k}\right) \rightarrow M O_{2}$, and wedging these maps together, one obtains a composite

$$
\tilde{g}: M^{n} \rightarrow \underset{k}{\bigvee} M O_{2}
$$

(Note. Brand's map is defined using only those terms $k \geqslant 2$, but here the wedge is for $k \geqslant 1$, not that it makes a significant difference.) One also has a composite

$$
g: N^{n} \stackrel{c}{\rightarrow} T(\nu) \rightarrow M O_{2}
$$

obtained by collapsing onto the tubular neighborhood of $B$ and then classifying. 
Beginning with the bundle $\gamma_{2}$ over $\mathrm{BO}_{2}$, one has maps

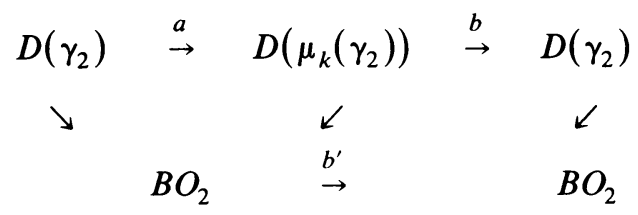

where $a$ is the degree $k$ wrapping on fibers and $b$ is the bundle map covering $b^{\prime}$ to classify $\mu_{k}\left(\gamma_{2}\right)$. The composite $b \circ a$ then induces a map $b \circ a: \mathrm{MO}_{2} \rightarrow \mathrm{MO}_{2}$ which may be wedged together to give a map $V_{k} M O_{2} \rightarrow M O_{2}$. If the tubular neighborhoods of the $\tilde{B}_{k}$ are taken as the inverse images of a small tubular nighborhood of $B$, one obtains a commutative diagram

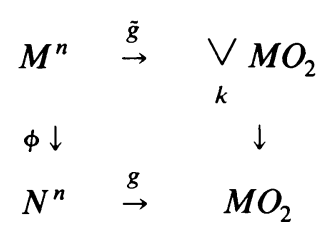

(up to homotopy of the classifying maps). (Note. This requires the wedge for $k \geqslant 1$.)

One then has a certain collection of cohomology classes. One has $U \in$ $H^{2}\left(M_{2} ; Z_{2}\right)$, the Thom class, and the Thom class $U_{k} \in H^{2}\left(V_{k} M_{2} ; Z_{2}\right)$ coming from the $k$ th wedge summand. Rather corrupting notation one has classes $w_{1}^{r} U$ and $w_{1}^{r} U_{k}$ obtained by applying the Thom isomorphism to $w_{1}^{r} \in H^{*}\left(B O_{2}\right)$. There is also a unique class $\mathfrak{p}_{1} \in H^{4}\left(M O_{2} ; Z\right)$ mapped to the Pontrjagin class $\mathfrak{p}_{1} \in H^{4}\left(B O_{2} ; Z\right)$ under the map $\mathrm{BO}_{2} \rightarrow \mathrm{MO}_{2}$ including the base space and one lets $\mathfrak{p}_{1, k} \in$ $\mathrm{H}^{4}\left(\mathrm{~V}_{k} \mathrm{MO}_{2} ; \mathrm{Z}\right)$ by taking the Pontrjagin class in the $k$ th wedge summand.

One then has the results of Brand [3]:

Proposition. One has

$$
w\left(\tau(M)-\phi^{*} \tau(N)\right)=1+\tilde{g}^{*}\left(\sum_{k \text { even }}\left(U_{k}+w_{1} U_{k}+w_{1}^{2} U_{k}+\cdots\right)\right) \in H^{*}\left(M^{n} ; Z_{2}\right)
$$

and

$$
\mathfrak{p}\left(\tau(M)-\phi^{*} \tau(N)\right)=1+\tilde{g}^{*}\left(\sum_{k} \sum_{l=1}^{\infty}(-1)^{l}\left(k^{2}-1\right) k^{2 l-2} \mathfrak{p}_{1, k}^{l}\right) \in H^{*}\left(M^{n} ; Z\right) .
$$

Note. Brand only refers to the classes $\mathfrak{p}_{1, k}$ in rational cohomology, and asserts the formula for the Pontrjagin class rationally. This all works integrally. If you consider the cofibration $\mathrm{BO}_{1} \stackrel{i}{\rightarrow} \mathrm{BO}_{2} \stackrel{j}{\rightarrow} M \mathrm{O}_{2}, \mathrm{H}^{*}\left(\mathrm{BO}_{1} ; Z\right)$ is isomorphic to the polynomial ring on the integral Bockstein of $w_{1}$ (of order 2), i.e. $Z\left[\beta w_{1}\right] /\left\{2 \beta w_{1}=0\right\}$ and $i^{*}\left(\beta w_{1}\right)=\beta w_{1}$ so $i^{*}$ is epic, and $j^{*}$ is monic. Since $i^{*}\left(p_{1}\right)=0$, there is a unique integral class hitting $\mathfrak{p}_{1}$. Using Brand's arguments one does the calculation by pulling back to $\mathrm{BO}_{2}$, where he uses the Whitney sum formula for Pontrjagin classes. Thus, the formula for the class $\mathfrak{p}\left(\tau(M)-\phi^{*} \tau(N)\right)$ is actually correct in integral cohomology modulo 2-torsion. To see that the formula is correct integrally one must 
check in the $\mathrm{BO}_{2}$ 's that the purported Pontrjagin class has the correct reduction to mod 2 cohomology and that is sufficient because all torsion in $\mathrm{H}_{*}\left(\mathrm{BO}_{2} ; \mathrm{Z}\right)$ has order 2. However, $\bmod 2 \Sigma_{k} \Sigma_{l=1}^{\infty}(-1)^{l}\left(k^{2}-1\right) k^{2 l-2} \mathfrak{p}_{1, k}^{l}$ is $\Sigma_{k \text { even }} \mathfrak{p}_{1, k}$ and has mod 2 reduction $\Sigma_{k \text { even }} U_{k}^{2}=\left(\Sigma_{k \text { even }} U_{k}\right)^{2}$.

One then has, almost trivially

Proposition 3. The set of classes $\left[M^{n}\right]-d\left[N^{n}\right]$ in $\mathfrak{R}_{n}$ for $d$-fold branched coverings of closed $n$-dimensional manifolds is

$$
\left\{\alpha \in \mathfrak{N}_{n} \mid w_{1}^{n}(\alpha)=0\right\}
$$

if $d \geqslant 2, n>0$.

Proof. If $\phi: M^{n} \rightarrow N^{n}$ is a $d$-fold branched cover, one has $w_{\omega}\left[\left[M^{n}\right]-d[N]\right]=$ $w_{\omega}[M]-d w_{\omega}[N]=w_{\omega}[M]-w_{\omega}(N)\left[\phi_{*}[M]\right]=\left(w_{\omega}(\tau(M))-\phi^{*} w_{\omega}(\tau N)\right)[M]$. Вy Brand's formula, $\phi^{*}\left(w_{1}(N)\right)=w_{1}(M)$ and so $w_{1}(M)^{n}[M]=\left(\phi^{*} w_{1}(N)\right)^{n}[M]$, and so $w_{1}^{n}\left[\left[M^{n}\right]-d\left[N^{n}\right]\right]=0$.

From [17, Proposition 9.2], a class $\alpha \in \mathfrak{N}_{n}$ with $w_{1}^{n}(\alpha)=0$ is the class of a manifold $M^{n}$ having an involution $T$ with fixed point set $F$ of codimension 2. Letting $\phi: M^{n} \rightarrow N^{n}=M^{n} / T$ be the quotient map, one has a branched covering of degree 2 with $\left[M^{n}\right]-2\left[N^{n}\right]=\left[M^{n}\right]=\alpha$. For $d>2$, let $\phi^{\prime}: M^{n} \cup(d-2) N^{n} \rightarrow N^{n}$ by using $\phi$ on $M$ and the trivial cover for $d-2$ copies of $N$ and then $\left[M^{n} \cup(d-2) N^{n}\right]-$ $d\left[N^{n}\right]=\left[M^{n}\right]-2\left[N^{n}\right]=\alpha$. Thus, obtains all classes $\alpha$ with $w_{1}^{n}(\alpha)=0$ from coverings.

Note. This trick of replacing a branched cover $\phi: M^{n} \rightarrow N^{n}$ by

$$
\phi^{\prime}: M^{n} \cup(d-\operatorname{deg} \phi) N^{n} \rightarrow N^{n}
$$

to increase the degree of the cover without changing the class $\left[M^{n}\right]-(\operatorname{deg} \phi)\left[N^{n}\right]$ will be used repeatedly.

Now consider

Proposition 2'. If $\phi: M^{n} \rightarrow N^{n}$ is a branched cover of closed manifolds, then $\left[M^{n}, \phi\right]-(\operatorname{deg} \phi)\left[N^{n}\right.$, identity $]$ in the bordism of $N$ is the class of the map

$$
R P\left(\tilde{\nu}_{\text {even }} \oplus 1\right) \rightarrow B \subset N .
$$

Proof. One considers a class $x \in H^{i}\left(N ; Z_{2}\right)$, and Stiefel-Whitney class $w_{\omega}$, and wishes to compute $w_{\omega} \phi^{*}(x)[M]-(\operatorname{deg} \phi) w_{\omega} x[N]=\left(w_{\omega} \phi^{*}(x)-\phi^{*}\left(w_{\omega}(N) \cdot x\right)\right)[M]$. For this one uses Brand's formula to write

$$
\begin{aligned}
w(M) & =w\left(\left(\tau(M)-\phi^{*} \tau(N)\right) \oplus \phi^{*} \tau(N)\right) \\
& =\phi^{*}(w(N))\left\{1+\sum_{k \text { even }}\left(U_{k}+w_{1} U_{k}+w_{1}^{2} U_{k}+\cdots\right)\right\},
\end{aligned}
$$

where notationally one deletes $\tilde{g}^{*}$. If one expands out $w_{\omega}(M)$ one obtains $\phi^{*}\left(w_{\omega}(N)\right)$ + terms involving factors $w_{i}^{r} U_{k}$, and the first term in that expression, when multiplied by $\phi^{*}(x)$ and evaluated on $[M]$ gives $\phi^{*}\left(w_{\omega}(N) \cdot x\right)[M]$. Thus the characteristic number remaining is the value on the fundamental class of $[M]$ of the part of $w_{\omega}(M) \phi^{*}(x)$ involving the classes $w_{1}^{r} U_{k}$. 
If you now consider $R P\left(\tilde{\nu}_{k} \oplus 1\right) \stackrel{\pi}{\rightarrow} \tilde{B}_{k} \stackrel{\phi}{\rightarrow} B \subset N$, and let $c \in H^{1}\left(R P\left(\tilde{\nu}_{k} \oplus 1\right) ; Z_{2}\right)$ be the first Stiefel-Whitney class of the double cover by the sphere bundle, one has

$$
w\left(R P\left(\tilde{\nu}_{k} \oplus 1\right)\right)=\pi^{*} w\left(\tilde{B}_{k}\right)\left\{(1+c)^{3}+w_{1}\left(\tilde{\nu}_{k}\right)(1+c)^{2}+w_{2}\left(\tilde{\nu}_{k}\right)(1+c)\right\}
$$

(where actually $w_{i}\left(\tilde{\nu}_{k}\right)$ should have a $\pi^{*}$ ) with a relation $c^{3}+w_{1}\left(\tilde{\nu}_{k}\right) c^{2}+w_{2}\left(\tilde{\nu}_{k}\right) c=0$. Also $\phi: \tilde{B}_{k} \rightarrow B$ is a covering so $\pi^{*} w\left(\tilde{B}_{k}\right)=\pi^{*} \phi^{*} w(B)=\pi^{*} \phi^{*}(w(N) / w(\nu))=$ $\phi^{*} w(N) / \pi^{*} \phi^{*} w(\nu)$, and assuming $k$ is even, $\phi^{*} w(\nu)=1+w_{1}\left(\tilde{\nu}_{k}\right)$, so

$$
\begin{aligned}
w\left(R P\left(\tilde{\nu}_{k} \oplus 1\right)\right) & =\phi^{*} w(N) \cdot\left\{\frac{(1+c)^{2}+w_{1}\left(\tilde{\nu}_{k}\right)(1+c)+w_{2}\left(\tilde{\nu}_{k}\right)}{1+w_{1}\left(\tilde{\nu}_{k}\right)}\right\}(1+c) \\
& =\phi^{*} w(N) \cdot\left\{\frac{1+w_{1}\left(\tilde{\nu}_{k}\right)+\left(c^{2}+w_{1}\left(\tilde{\nu}_{k}\right) c+w_{2}\left(\tilde{\nu}_{k}\right)\right)}{1+w_{1}\left(\tilde{\nu}_{k}\right)}\right\}(1+c) \\
& =\phi^{*} w(N) \cdot\left\{1+U_{k}+w_{1}\left(\tilde{\nu}_{k}\right) U_{k}+w_{1}\left(\tilde{\nu}_{k}\right)^{2} U_{k}+\cdots\right\}(1+c),
\end{aligned}
$$

where $U_{k}=c^{2}+w_{1}\left(\tilde{\nu}_{k}\right) c+w_{2}\left(\tilde{\nu}_{k}\right)$.

Note. One has a cofibration $R P\left(\tilde{\nu}_{k}\right) \rightarrow R P\left(\tilde{\nu}_{k} \oplus 1\right) \rightarrow T\left(\tilde{\nu}_{k}\right)$ and $U_{k}$ is the pull back of the Thom class in $T\left(\tilde{\nu}_{k}\right)$. It is the "same" class as the Brand class, but considered in a different space. In homology, $M \rightarrow \bigvee_{k \text { even }} T\left(\tilde{\nu}_{k}\right)$ sends the fundamental class of $M$ to the same class as the image of the fundamental class under

$$
\bigcup_{k \text { even }} R P\left(\tilde{\nu}_{k} \oplus 1\right) \rightarrow \underset{k \text { even }}{\bigvee} T\left(\tilde{\nu}_{k}\right)
$$

Define

$$
\begin{aligned}
\hat{w}\left(R P\left(\tilde{\nu}_{k} \oplus 1\right)\right) & =w\left(R P\left(\tilde{\nu}_{k} \oplus 1\right)\right) /(1+c) \\
& =\phi^{*} w(N)\left\{1+U_{k}+w_{1}\left(\tilde{\nu}_{k}\right) U_{k}+w_{1}\left(\tilde{\nu}_{k}\right)^{2} U_{k}+\cdots\right\} .
\end{aligned}
$$

Noting that the evaluation of $\hat{w}_{\omega}\left(R P\left(\tilde{\nu}_{k} \oplus 1\right)\right) \phi^{*}(x)\left[R P\left(\tilde{v}_{k} \oplus 1\right)\right]$ annihilates the term $\phi^{*} w_{\omega}(N) \phi^{*}(x)$, which comes from $H^{n}\left(\tilde{B}_{k} ; Z_{2}\right)=0$, only the terms involving classes $w_{1}\left(\tilde{\nu}_{k}\right)^{r} U_{k}$ give nonzero value. One then has

OBSERVATION. $w_{\omega} \phi^{*}(x)[M]-(\operatorname{deg} \phi) w_{\omega} x[N]=\hat{w}_{\omega} \phi^{*}(x)\left[R P\left(\tilde{\nu}_{\text {even }} \oplus 1\right)\right]$, where

$$
\hat{w}=w\left(R P\left(\tilde{\nu}_{\text {even }} \oplus 1\right)\right) /(1+c) \text {. }
$$

Of course, what this means is that one calculates with the class $\hat{w}$ just as if it were a Stiefel-Whitney class and one was computing a Stiefel-Whitney number. To complete the proof of the proposition one has

Lemma. Let $N$ be a space and $f: P^{n} \rightarrow N, g: Q^{n} \rightarrow N$ two maps of closed manifolds into $N$. Suppose there is a class $c \in H^{1}\left(P ; Z_{2}\right)$ and that $\hat{w}=w(P) /(1+c)$. If for all $x \in H^{i}\left(N ; Z_{2}\right)$ and all $\omega$ one has

$$
\hat{w}_{\omega} f^{*}(x)[P]=w_{\omega} g^{*}(x)[Q],
$$

then $\left[P^{n}, f\right]=\left[Q^{n}, g\right]$ in $\Re_{n}(N)$; i.e. $\hat{w}_{\omega} f^{*}(x)[P]=w_{\omega} f^{*}(x)[P]$.

Note. What this says is that if the modified Stiefel-Whitney number of a bordism element is again a bordism element, then the modification was irrelevant. The 
modification simply does not give the characteristic numbers of a bordism element in general.

Proof. Let $\bar{w}=1 / w, \overline{\hat{w}}=1 / \hat{w}$ for the dual Stiefel-Whitney classes. One then has

$$
\left\langle\overline{\hat{w}}_{i} \hat{w}_{\omega} f^{*}(x),[P]\right\rangle=\left\langle\bar{w}_{i} w_{\omega} g^{*}(x),[Q]\right\rangle=\left\langle\chi\left(\mathrm{Sq}^{i}\right) w_{\omega} g^{*}(x),[Q]\right\rangle,
$$

and

$$
\begin{aligned}
\chi\left(\mathrm{Sq}^{i}\right) \hat{w}_{\omega} f^{*}(x) & =\sum_{j} \chi\left(\mathrm{Sq}^{j}\right)\left(\hat{w}_{\omega}\right) f^{*}\left(\chi\left(\mathrm{Sq}^{i-j}\right) x\right) \\
& =\sum_{j}\left(\sum_{\omega^{\prime}} a_{\omega^{\prime}}^{\omega, j} \hat{w}_{\omega^{\prime}}\right) f^{*}\left(\chi\left(\mathrm{Sq}^{i-j}\right) x\right)
\end{aligned}
$$

where $\chi\left(\mathrm{Sq}^{j}\right)\left(\hat{w}_{\omega}\right)=\sum_{\omega^{\prime}} a_{\omega^{\prime}}^{\omega, j} \hat{w}_{\omega^{\prime}}$ is the universal formula for the Steenrod operation on a Stiefel-Whitney class of a bundle, and $\hat{w}=w(\tau(P)-l)$ where $w_{1}(l)=c$ is the class of a bundle, so

$$
\begin{aligned}
\left\langle\chi\left(\mathrm{Sq}^{i}\right) \hat{w}_{\omega} f^{*}(x),[P]\right\rangle & =\left\langle\sum_{j}\left(\sum_{\omega} a_{\omega^{\prime}}^{\omega, j} \hat{w}_{\omega^{\prime}}\right) f^{*}\left(\chi\left(\mathrm{Sq}^{i-j}\right) x\right),[P]\right\rangle \\
& =\left\langle\sum_{j}\left(\sum_{\omega} a_{\omega^{\prime}}^{\omega, j} w_{\omega^{\prime}}\right) g^{*}\left(\chi\left(\mathrm{Sq}^{i-j}\right) x\right),[Q]\right\rangle \\
& =\left\langle\chi\left(\mathrm{Sq}^{i}\right) w_{\omega} g^{*}(x),[Q]\right\rangle .
\end{aligned}
$$

Thus one has

$$
\left\langle\overline{\hat{w}}_{i} \hat{w}_{\omega} f^{*}(x),[P]\right\rangle=\left\langle\chi\left(\mathrm{Sq}^{i}\right) \hat{w}_{\omega} f^{*}(x),[P]\right\rangle=\left\langle\bar{w}_{i} \hat{w}_{\omega} f^{*}(x),[P]\right\rangle
$$

or

$$
\left\langle\left(\overline{\hat{w}}_{i}+\bar{w}_{i}\right) \hat{w}_{\omega} f^{*}(x),[P]\right\rangle=0 .
$$

Summing over all $i$, one has

$$
\left\langle(\overline{\hat{w}}+\bar{w}) \hat{w}_{\omega} f^{*}(x),[P]\right\rangle=0 .
$$

Noting that $\hat{w}=w /(1+c), w=\hat{w}(1+c)$ so

$$
\bar{w}=\overline{\hat{w}}\left(1+c+c^{2}+c^{3}+\cdots\right)
$$

and

$$
\overline{\hat{w}}+\bar{w}=\overline{\hat{w}}\left(c+c^{2}+c^{3}+\cdots\right) .
$$

Thus, one has

$$
\left\langle\left(c+c^{2}+c^{3}+\cdots\right) \hat{w} \hat{w}_{\omega} f^{*}(x),[P]\right\rangle=0
$$

for all $\omega$ and $x$, so that powers of $c$ annihilate all expressions $w_{\omega} f^{*}(x)$ when evaluated on $[P]$. Since $w=\hat{w}(1+c), w_{\omega}=\hat{w}_{\omega}+\sum_{i>0} c^{l} \cdot b_{\omega^{\prime}}^{\omega} \hat{w}_{\omega^{\prime}}$ in a universal formula, and so

$$
\begin{aligned}
\left\langle w_{\omega} f^{*}(x),[P]\right\rangle & =\left\langle\left(\hat{w}_{\omega}+\sum_{i>0} c^{i} b_{\omega^{\prime}}^{\omega} \hat{w}_{\omega^{\prime}}\right) f^{*}(x),[P]\right\rangle \\
& =\left\langle\hat{w}_{\omega} f^{*}(x),[P]\right\rangle=\left\langle w_{\omega} g^{*}(x),[Q]\right\rangle .
\end{aligned}
$$

Thus, the maps $f$ and $g$ have the same Stiefel-Whitney numbers. 
Special Note. If one reverses this, one sees that $c^{i} w_{\omega} \phi^{*}(x)\left[R P\left(\tilde{\nu}_{\text {even }} \oplus 1\right)\right]=0$, which is equivalent to the assertion that $R P\left(\tilde{\nu}_{\text {even }}\right) \rightarrow N \times R P^{\infty}$, with the map induced by $\phi$ and the class $c$, is cobordant to zero. For involutions, this is a crucial feature of Conner and Floyd's work with involutions [6, (24.1)] and is the observation $S(\tilde{\nu}) \rightarrow N$ freely bounds $M$-interior $(D(\tilde{\nu})) \stackrel{\phi}{\rightarrow} N$. The above argument shows that the analogue holds for branched covers, but this is certainly not a direct geometric argument.

REMARK. These results do not agree with Theorem 3.2 of [2], which is valid only with the additional unstated hypothesis that $\left.w(N)\right|_{B_{\phi}}=1$. In line 1 of the proof, $\bar{w}\left(B_{\phi}\right)$ is the normal class of $B_{\phi}$ in $N$, while on line 4 it is the normal class in Euclidean space. In the applications only this special case was used. With the hypotheses given the correct conclusion is $\left.w(M)\right|_{\phi^{-1} B_{\phi}}=\left.\phi^{*} w(N)\right|_{\phi^{-1} B_{\phi}}$. One should also remark that the hypothesis that $M^{n}$ have even Euler characteristic is unnecessary in Corollary 3.5 of [2], since $w_{n}\left(M^{n}\right)=\left(v_{n / 2}\left(M^{n}\right)\right)^{2}$ and is also a product, where $v$ is the $\mathrm{Wu}$ class.

COROllary. If $\phi: M^{n} \rightarrow N^{n}$ is a branched covering of closed manifolds with $w_{1}\left(\tilde{\nu}_{\text {even }}\right) \in \operatorname{image}\left\{i^{*} \phi^{*}: H^{*}\left(N^{n}, Z_{2}\right) \rightarrow H^{*}\left(\tilde{B}_{\text {even }} ; Z_{2}\right)\right.$ then $\left[M^{n}\right]-(\operatorname{deg} \phi)\left[N^{n}\right]=$ 0 in $\mathfrak{N}_{*}$.

Note. This condition is satisfied if $B_{\phi}$ is orientable, if $\tilde{B}_{\text {even }}$ is orientable, if $\nu$ is orientable, or $\tilde{\nu}_{\text {even }}$ is orientable, for one has either $w_{1}\left(\tilde{\nu}_{\text {even }}\right)=i^{*} \phi^{*} w_{1}(N)$ or $w_{1}\left(\tilde{\nu}_{\text {even }}\right)$ $=i^{*} \phi^{*}(0)$. In particular, Theorem (4.4) of Hattori [8] is a special case of this.

Proof. As noted above, $(\phi \circ \pi) \times c: R P\left(\tilde{\nu}_{\text {even }}\right)^{\circ} \rightarrow N \times B Z_{2}$ bounds, and

$$
\begin{aligned}
w\left(R P\left(\tilde{\nu}_{\text {even }}\right)\right) & =w\left(\tilde{B}_{\text {even }}\right)\left\{(1+c)^{2}+w_{1}\left(\tilde{\nu}_{\text {even }}\right)(1+c)+w_{2}\left(\tilde{\nu}_{\text {even }}\right)\right\} \\
& =w\left(\tilde{B}_{\text {even }}\right)\left\{1+w_{1}\left(\tilde{\nu}_{\text {even }}\right)\right\}
\end{aligned}
$$

with $c^{2}+c w_{1}\left(\tilde{\nu}_{\text {even }}\right)+w_{2}\left(\tilde{\nu}_{\text {even }}\right)=0$. Letting $w_{1}\left(\tilde{\nu}_{\text {even }}\right)=\phi^{*}(x)$, one has for any $i, j$, $\omega$ that

$$
\begin{aligned}
0 & =c\left\{c^{2}+c \phi^{*}(x)\right\}^{i} \phi^{*}(x)^{j} \cdot\left(\frac{w\left(R P\left(\tilde{\nu}_{\text {even }}\right)\right)}{1+\phi^{*}(x)}\right)_{\omega}\left[R P\left(\tilde{\nu}_{\text {even }}\right)\right] \\
& =c w_{2}\left(\tilde{\nu}_{\text {even }}\right)^{i} w_{1}\left(\tilde{\nu}_{\text {even }}\right)^{j} w_{\omega}\left(\tilde{B}_{\text {even }}\right)\left[R P\left(\tilde{\nu}_{\text {even }}\right)\right] \\
& =w_{2}\left(\tilde{\nu}_{\text {even }}\right)^{i} w_{1}\left(\tilde{\nu}_{\text {even }}\right)^{j} w_{\omega}\left(\tilde{B}_{\text {even }}\right)\left[\tilde{B}_{\text {even }}\right]
\end{aligned}
$$

and hence the map $\tilde{\nu}_{\text {even }}: \tilde{B}_{\text {even }} \rightarrow B O_{2}$ bounds, and so $R P\left(\tilde{\nu}_{\text {even }} \oplus 1\right)$ bounds.

Note. By including a factor $\phi^{*}(y)$ with $y \in H^{*}\left(N ; Z_{2}\right)$ one may conclude that $(\phi \circ i) \times \tilde{\nu}_{\text {even }}: \tilde{B}_{\text {even }} \rightarrow N \times B O_{2}$ bounds to see that $\left[M^{n}, \phi\right]-(\operatorname{deg} \phi)[N$, identity] $=0$ in $\mathfrak{N}_{n}\left(N^{n}\right)$.

4. Oriented branched covers. To begin the study of the oriented case, one has

LEMMA 1. Every class $\alpha \in \operatorname{Tor}\left(\Omega_{n}\right)$ is of the form $\left[M^{n}\right]-(\operatorname{deg} \phi)\left[N^{n}\right]$ for some branched covering of closed oriented manifolds of degree $d$, if $d \geqslant 2$. 
Proof. One has a homomorphism $\partial: \mathfrak{R}_{n+1} \rightarrow \Omega_{n}$ assigning to $P^{n+1}$ the class of the submanifold dual to $w_{1}$. According to Wall [18], $\partial$ maps onto Tor $\left(\Omega_{n}\right)$ and in fact $\partial$ : $\mathcal{w}_{n+1} \rightarrow \Omega_{n}$ maps onto the torsion where $\mathcal{Q W}_{n+1}$ is the cobordism group of manifolds with $w_{1}$ reduced integral.

Being given $d \geqslant 2$ and $\alpha \in \operatorname{Tor}\left(\Omega_{n}\right)$, there is a class $\beta \in \mathfrak{N}_{n+1}$ having all numbers divisible by $w_{1}^{2}$ zero, i.e., coming from $\mho_{n+1}$, and so that $\partial \beta=\alpha$. By Proposition 3 , there is a branched covering $\theta: P^{n+1} \rightarrow Q^{n+1}$ of degree $d$ for which $[P]-d[Q]=\beta$. Let $f: Q^{n+1} \rightarrow R P^{N}$ for some large integer $N$ with $f^{*}(i)=w_{1}(Q)$ where $i \in$ $H^{1}\left(R P^{N} ; Z_{2}\right)$ is the nonzero class, and deform $\left.f\right|_{B_{\theta}}$ to be transverse to $R P^{N-1}$ and then $\operatorname{deform} f$ to be the projection

$$
D(\nu) \rightarrow B_{\theta} \stackrel{\left(\left.f\right|_{B_{\theta}}\right)^{\prime}}{\rightarrow} R P^{N}
$$

on a tubular neighborhood of $B_{\theta} . f$ is then transverse to $R P^{N-1}$ on a neighborhood of $B_{\theta}$ and without changing the map on a smaller tubular neighborhood of $B_{\theta}$ one may further deform $f$ to be transverse to $R P^{N-1} \subset R P^{N}$. Thus, one assumes $f$ has this form; i.e. $f$ and $\left.f\right|_{B_{\theta}}$ are transverse to $R P^{N-1}$ and on a tubular neighborhood of $B_{\theta}, f$ is given by projection on $B_{\theta}$ followed by $\left.f\right|_{B_{\theta}}$. The composite $f \circ \theta$ is then also transverse to $R P^{N-1}$ with $\left.f \circ \theta\right|_{\theta^{-1} B_{\theta}}$ being transverse to $R P^{N-1}$ and being given by $(f \circ \theta) \circ$ projection on a tubular neighborhood of $\theta^{-1} B_{\theta}$, and further $(f \circ \theta)^{*}(i)=$ $\theta^{*}\left(w_{1}(Q)\right)=w_{1}(P)$.

Letting $\bar{P}^{n} \subset P^{n+1}$ and $\bar{Q}^{n} \subset Q^{n+1}$ be $(f \circ \theta)^{-1}\left(R P^{N-1}\right)$ and $f^{-1}\left(R P^{N-1}\right), \bar{\theta}$ : $\bar{P}^{n} \rightarrow \bar{Q}^{n}$ is then a branched covering of degree $d$, where $\bar{\theta}=\left.\theta\right|_{\bar{P}^{n}}$. Further $\bar{P}$ and $\bar{Q}$ are orientable, being the duals to $w_{1}$ and one has $[\bar{P}]-d[\bar{Q}]=(\partial[P]-d \partial[Q])=$ $\partial([P]-d[Q])=\partial \beta=\alpha$. (Note. Identification of the normal bundle of $\bar{Q}$ in $Q$ with det $\tau(Q) \mid \bar{Q}$ and similarly for $\bar{P}$ gives a choice of compatible orientations by the $\partial$ process. The only indeterminancy is to completely reverse orientation in the $\partial$ process, i.e. $\bar{Q} \cong-\bar{Q}$, which does not change $\alpha$.) Thus the class $\alpha$ is represented in the desired form.

This reduces the problem of realizing classes in $\Omega_{*}$ entirely to a question of possible Pontrjagin numbers and the realization of classes in $\Omega_{*} / \operatorname{Tor}\left(\Omega_{*}\right)$.

Lemma 2. Let $n=4 m$ and $s_{m}(\mathfrak{p})$ the primitive Pontrjagin class. If $\phi: M^{n} \rightarrow N^{n}$ is a branched covering of closed oriented manifolds, then

$$
\begin{aligned}
s_{m}(\mathfrak{p})\left[\left[M^{n}\right]-(\operatorname{deg} \phi)\left[N^{n}\right]\right] & =\sum_{k \geqslant 2}\left(1-k^{2 m}\right) \mathfrak{p}_{1, k}^{m}\left[M^{n}\right] \\
& =\sum_{k \geqslant 2}\left(1-k^{2 m}\right) \mathfrak{p}_{1}\left(\tilde{v}_{k}\right)^{m-1}\left[\tilde{B}_{k} \cap \tilde{B}_{k}\right] .
\end{aligned}
$$

Proof. Clearly,

$$
\begin{aligned}
s_{m}(\mathfrak{p})\left[\left[M^{n}\right]-(\operatorname{deg} \phi)\left[N^{n}\right]\right] & =s_{m}(\mathfrak{p})\left[M^{n}\right]-(\operatorname{deg} \phi) s_{m}(\mathfrak{p})\left[M^{n}\right] \\
& =\left\{s_{m}(\mathfrak{p})(\tau(M))-\phi^{*} s_{m}(\mathfrak{p})(\tau(N))\right\}\left[M^{n}\right] \\
& =\left\{s_{m}(\mathfrak{p})\left(\tau(M)-\phi^{*} \tau(N)\right)\right\}\left[M^{n}\right]
\end{aligned}
$$


by primitivity. To compute this class universally, following Brand [4], one has $\mathfrak{p}=\left(1+\mathfrak{p}_{1}\right) /\left(1+k^{2} \mathfrak{p}_{1}\right) \in H^{*}\left(B O_{2} ; Z\right)$ to give $s_{m}(\mathfrak{p})=\mathfrak{p}_{1}^{m}-k^{2 m} \mathfrak{p}_{1}^{m}=$ $\left(1-k^{2 m}\right) \mathfrak{p}_{1}^{m}$. The rest of the result is the observation that $\tilde{B}_{k} \cap \tilde{B}_{k}$ is the submanifold dual to $\mathfrak{p}_{1, k}$, and $\mathfrak{p}_{1, k} \mid \tilde{B}_{k} \cap \tilde{B}_{k}=\mathfrak{p}_{1}\left(\tilde{\nu}_{k}\right)$.

Combining results, one then has easily

Proposition 4'. The set classes $\alpha \in \Omega_{n}$ of the form $\left[M^{n}\right]-2\left[N^{n}\right]$ with $\phi: M^{n} \rightarrow N^{n}$ a degree 2 branched covering of closed oriented manifolds is a subgroup of $\Omega_{n}$ of odd index, if $n>0$.

Proof. If $\phi: M^{n} \rightarrow N^{n}$ is a degree 2 branched covering so is $\phi \times$ (identity): $M^{n} \times P^{m} \rightarrow N^{n} \times P^{m}$, and so the set of classes $\alpha$ of the form $\left[M^{n}\right]-2\left[N^{n}\right]$ in $\Omega_{*}$ forms an ideal $\Lambda_{*}$ (i.e. $\Omega_{*}$ submodule).

By Proposition $1^{\prime \prime}, \Omega_{n}^{Z_{2}}(\operatorname{cod} 2) \cong \Omega_{n} \oplus \Omega_{n-4}\left(B O_{2}\right)$ and one has $\left[\xi^{2} \rightarrow \mathbf{C} P^{2 r}\right] \in$ $\Omega_{4 r}\left(B O_{2}\right)$, with $\xi^{2}$ the Hopf bundle, having $\mathfrak{p}_{1}^{r}\left(\xi^{2}\right)\left[C P^{2 r}\right]=1$. Since this is the cobordism class of the self-intersection $\left[\tilde{\nu}_{2} \rightarrow \tilde{B}_{2} \cap \tilde{B}_{2}\right]$ for some 2-fold branched cover $\theta: P^{4 r+4} \rightarrow Q^{4 r+4}$, one has a class $\alpha=\left[P^{4 r+4}\right]-2\left[Q^{4 r+4}\right] \in \Lambda_{4 r+4}$ for which $s_{r+1}(\mathfrak{p})[\alpha]=\left(1-2^{2 r+2}\right)$. This $\alpha$ is a suitable polynomial generator for $\left(\Omega_{*} /\right.$ Tor $\left.\Omega_{*}\right)$ $\otimes Z_{2}$.

Since $\Lambda_{*}$ contains Tor $\Omega_{*}$ and maps onto $\left(\Omega_{*} /\right.$ Tor $\left.\Omega_{*}\right) \otimes Z_{2}$ in positive dimensions, $\Lambda_{n} \subset \Omega_{n}$ has odd index if $n>0$.

REMARK. One may actually write down branched coverings for the low dimensional classes in $\Omega_{*}$. Specifically, one has $\pi: \mathbf{C} P^{2} \rightarrow \mathbf{C} P^{2} /$ conjugation $\cong S^{4}$, with the identification to $S^{4}$ due to Kuiper [10], for which the self-intersection class is the inclusion of a point in $B O_{2}$. One also has $\pi: P(1,2) \rightarrow S^{1} \times S^{4}$, where $P(1,2)=S^{1}$ $\times \mathbf{C P}(2) /(-1 \times$ conjugation $)$ is the Dold manifold and $\pi$ is the quotient by dividing out the involution $-1 \times 1 \sim 1 \times$ conjugation, with the self-intersection being the nonzero element in $\Omega_{1}\left(B O_{2}\right) \cong Z_{2}$. From $\S 2$, one also has the branched covering $\phi: Q^{2 r} \rightarrow \mathbf{C} P^{2 r}$, where $Q^{2 r}$ is the quadric for which $\left[Q^{2 r}\right]-2\left[\mathbf{C} P^{2 r}\right]$ is $2\left\{\left[H P^{r}\right]-\left[C P^{2 r}\right]\right\}$. Since an odd multiple of this class may also be hit, one has $\left[H P^{r}\right]-\left[C P^{2 r}\right] \in \Lambda_{4 r}$, and this class has $s$-number $1-2^{2 r}$, to give an explicit choice of generators for $\Lambda_{*}$.

Proposition 5'. If $n=4 m=2 k(p-1)$ with $p$ an odd prime and $\alpha=\left[M^{n}\right]-$ $d\left[N^{n}\right]$ is the class of a $d$-fold branched cover with $d<2 p$, then $s_{m}(\mathfrak{p})[\alpha] \equiv 0 \bmod p$.

Proof. Let $\phi: M^{n} \rightarrow N^{n}$ be the branched covering. By Lemma 2, $s_{m}(\mathfrak{p})[\alpha]=$ $\Sigma_{j \geqslant 2}\left(1-j^{2 m}\right) \mathfrak{p}_{1, j}^{m}\left[M^{n}\right]$ where the sum is for $j \leqslant d$ only. For $j \neq 0, j^{2 m}=j^{k(p-1)} \equiv$ $1 \bmod p$, and hence $\bmod p, s_{m}(\mathfrak{p})[\alpha] \equiv \mathfrak{p}_{1, p}^{m}\left[M^{n}\right]=\mathfrak{p}_{1}^{m-1}\left(\tilde{\nu}_{p}\right)\left[\tilde{B}_{p} \cap \tilde{B}_{p}\right]$. If one considers a component $\tilde{B}_{p}^{i}=\bar{B}$ of $\tilde{B}_{p}$, with $B^{\prime}=\phi \bar{B}$ being the corresponding component of the branch set with $\phi: \bar{B} \rightarrow B^{\prime}$ being an $r$-fold cover, $2 p>d \geqslant r \cdot p$ and hence $r=1$, and $\phi: \bar{B} \rightarrow B^{\prime}$ is an isomorphism. If $\bar{\nu}$ and $\nu^{\prime}$ are the normal bundles, then $\nu^{\prime} \cong \mu_{p}(\bar{\nu})$ and $\phi: S(\bar{\nu}) \rightarrow S\left(\nu^{\prime}\right)$ is a $p$-fold cover, forming a portion of the $d$-fold covering $\phi: \phi^{-1}\left(S\left(\nu^{\prime}\right)\right) \rightarrow S\left(\nu^{\prime}\right)$ classified by a map $S\left(\nu^{\prime}\right) \stackrel{f}{\rightarrow} B \Sigma_{d}$. This local covering actually factors through a map $S\left(\nu^{\prime}\right) \rightarrow B \Sigma_{d-p} \times B \Sigma_{p}$ corresponding to the two parts of the covering $\phi^{-1}\left(S\left(\nu^{\prime}\right)\right)-S(\bar{\nu})$ and $S(\bar{\nu})$. 
Claim. The number $\mathfrak{p}_{p}^{m-1}(\bar{\nu})[\bar{B} \cap \bar{B}] \in Z_{p}$ is precisely the class $f_{*}\left[S\left(\nu^{\prime}\right)\right] \in$ $p$-torsion part of $H_{2 k(p-1)-1}\left(B \Sigma_{d} ; Z\right)=Z_{p}$.

Since the local branchings for $\phi: M^{n} \rightarrow N^{n}$ give rise to the zero element in $\Omega_{n-1}\left(B \Sigma_{d}\right)$, the total homology class in the $p$-torsion part of $H_{n-1}\left(B \Sigma_{d} ; Z\right)$ is zero, and hence one must have $\mathfrak{p}_{1}\left(\tilde{\nu}_{p}\right)^{m-1}\left[\tilde{B}_{p} \cap \tilde{B}_{p}\right]=0$.

Note. For the necessary information about $H_{*}\left(B \Sigma_{d} ; Z\right)$, one should recall the work of Nakaoka $[13,14]$.

There are undoubtedly many ways to see this claim, and one rather unsophisticated way is to consider the diagram,

$$
\begin{aligned}
& \Omega_{*-4}\left(B S O_{2}\right) \stackrel{b}{\leftarrow} \tilde{\Omega}_{*}\left(M S O_{2}\right) \stackrel{a}{\rightarrow} \Omega_{*-1}\left(B Z_{p}\right) \quad \rightarrow \quad H_{*}\left(B Z_{p} ; Z\right) \\
& \downarrow c \quad \downarrow \quad \downarrow \quad \downarrow e \\
& \Omega_{*-4}\left(B O_{2}\right) \stackrel{d}{\leftarrow} \tilde{\Omega}_{*}\left(M O_{2}\right) \quad \rightarrow \quad \Omega_{*-1}\left(B \Sigma_{p}\right) \quad \rightarrow \quad H_{*}\left(B \Sigma_{p} ; Z\right) \\
& \downarrow \\
& \Omega_{*-1}\left(B \Sigma_{d}\right) \quad \rightarrow \quad H_{*}\left(B \Sigma_{d} ; Z\right)
\end{aligned}
$$

where, for a reduced bordism element, the map to the left takes the self-intersection and to the right one takes the $p$-fold ramified cover or local branching, and one takes the usual maps from bordism to homology and the maps of classifying spaces induced by the inclusion of $Z_{p}$, the Sylow $p$ subgroup, in $\Sigma_{p}$ and $\Sigma_{d}$, with any two inclusions being conjugate.

Now $\tilde{\Omega}_{*}\left(\mathrm{MSO}_{2}\right) \cong \Omega_{*-2}\left(\mathrm{BSO}_{2}\right)$ is the free $\Omega_{*}$ module on the classes $\mathrm{CP}^{r} \rightarrow \mathrm{BSO}_{2}$ classifying the Hopf bundle. Applying $a$ takes the class of $\left[S^{2 r+1}, \exp (2 \pi i / p)\right]$ as free $Z_{p}$ action or the map of the standard lens space $L^{2 r+1}(p)$ into $B Z_{p}$, giving the standard generator in $H_{2 r+1}\left(B Z_{p} ; Z\right)=Z_{p}$. Further, decomposables in the $\Omega_{*}$ module structure of $\tilde{\Omega}_{*}\left(\mathrm{MSO}_{2}\right)$ give zero in homology and hence for $V^{2 r+2} \rightarrow \mathrm{MSO}_{2}$, $U_{2}^{r+1}[V, \partial V] \in Z_{p}$ is just the multiple of the standard generator in homology which is hit by the class of $V$. Now $U_{2}^{r+1}[V, \partial V]=X^{r+1}[B]$ where $X$ is the Euler class in $\mathrm{H}^{2}\left(\mathrm{BSO}_{2} ; \mathrm{Z}\right)$ and $\mathrm{B} \rightarrow \mathrm{BSO}_{2}$ is obtained by applying $\mathrm{b}$ to the class of $\mathrm{V} \rightarrow \mathrm{MSO}_{2}$, i.e. taking the appropriate self-intersection.

If one ignores the prime $2, d$ is an isomorphism, for the third term in the exact sequence with $d$ is $\tilde{\Omega}_{*}\left(\Sigma M O_{1}\right) \cong \mathfrak{N}_{*-2}$ which is a 2 group. Also ignoring the prime 2 and taking $* \equiv 0 \bmod 4, c$ becomes an isomorphism. (Ignoring $2, \Omega_{*}$ is entirely concentrated in dimensions a multiple of 4 and the $\mathrm{CP} P^{2 r} \rightarrow \mathrm{BO}_{2}$ form a base of $\Omega_{*}\left(\mathrm{BO}_{2}\right)$ ignoring 2. Similarly, the $\mathrm{CP} \mathrm{P}^{2 r} \rightarrow \mathrm{BSO}_{2}$ form an $\Omega_{*}$ base for $\Omega_{4 *}\left(\mathrm{BSO}_{2}\right)$ and the $\mathrm{CP}^{2 r+1} \rightarrow \mathrm{BSO}_{2}$ form an $\Omega_{*}$ base for $\Omega_{4 *+2}\left(\mathrm{BSO}_{2}\right)$.)

Commutativity of the diagram then gives the claim, since $e$ and $f$ are epimorphisms on the $p$-primary part of the homology.

One may obtain fairly precise information about the possible $s$-numbers with

Proposition. The set of possible s-numbers $s_{m}(\mathfrak{p})\left[\left[M^{4 m}\right]-d\left[N^{4 m}\right]\right]$ for $d$-fold branched coverings of closed oriented manifolds is the subgroup $s_{m}^{d} Z$ of the integers where

$$
s_{m}^{d}=a \cdot \operatorname{gcd}\left\{\left(1-2^{2 m}\right),\left(1-3^{2 m}\right), \ldots,\left(1-d^{2 m}\right)\right\}
$$


and $a=p_{1} \cdot p_{2} \cdots p_{r}, p_{1}<p_{2}<\cdots<p_{r}$, is a product of odd primes with $p_{i} \leqslant d$ and $p_{i}-1$ dividing $2 m$. If $p$ is an odd prime with $p \leqslant d$ and $p-1$ dividing $2 m$, then $p$ occurs in $a$ if either $2 m+1$ is a power of $p$ or $d<2 p$.

Proof. By taking the disjoint union of $d$-fold covers and by reversing orientation one sees that the set of $s_{m}(\mathfrak{p})[\alpha]$ forms a subgroup of $Z$, and so is $s_{m}^{d} Z$ for some integer $s_{m}^{d}$.

$$
\begin{aligned}
& \text { Let } h_{m}^{d}=\operatorname{gcd}\left\{\left(1-2^{2 m}\right),\left(1-3^{2 m}\right), \ldots,\left(1-d^{2 m}\right)\right\}, \text { and } \\
& \qquad g_{m}^{d}=\operatorname{gcd}\left\{\left(1-2^{2 m}\right), 3\left(1-3^{3 m}\right), \ldots, d\left(1-d^{2 m}\right)\right\} .
\end{aligned}
$$

For any $d$-fold branched cover, Lemma 2 gives

$$
s_{m}(\mathfrak{p})[\alpha]=\sum_{d \geqslant k \geqslant 2}\left(1-k^{2 m}\right) \mathfrak{p}_{1, k}^{m}\left[M^{4 m}\right]
$$

and since each $\mathfrak{p}_{1, k}^{m}\left[M^{4 m}\right]$ is integral, $h_{m}^{d}$ divides $s_{m}(\mathfrak{p})[\alpha]$, and hence $h_{m}^{d} \mid s_{m}^{d}$. One also has a 2-fold, and hence $d$-fold, branched covering with $s_{m}(\mathfrak{p})[\alpha]=\left(1-2^{2 m}\right)$, and for $j \leqslant d$ one has the $j$-fold covering $Q_{j}^{2 m} \rightarrow \mathbf{C} P^{2 m}$ by the $j$-dric having $s_{m}(\mathfrak{p})[\alpha]=j\left(1-j^{2 m}\right)$, hence also a $d$-fold branched covering with the same number. Thus $s_{m}^{d}$ divides $\left(1-2^{2 m}\right)$ and $j\left(1-j^{2 m}\right)$ for $3 \leqslant j \leqslant d$, and hence their greatest common divisor, so $s_{m}^{d}$ divides $g_{m}^{d}$.

Now, for $p$ dividing $h_{m}^{d}$, one has $p>d$ since for $p \leqslant d, p$ does not divide $1-p^{2 m}$. Then $p$ divides $g_{m}^{d}$ and if $p^{r}$ is the power of $p$ dividing $g_{m}^{d}$, then $p^{r} \mid j\left(1-j^{2 m}\right)$ and $j \leqslant d<p, p \nmid j$ so $p \mid\left(1-j^{2 m}\right)$, and so $p^{r}$ divides $h_{m}^{d}$. Thus $g_{m}^{d}=b h_{m}^{d}$ where $b$ is divisible only by primes less than or equal to $d$ and $h_{m}^{d}$ only by primes larger than $d$.

For $p \leqslant d, p^{2}$ is not a factor of $p\left(1-p^{2 m}\right)$ and so $b$ cannot be divisible by $p^{2}$. If $p-1$ divides $2 m$, then for $j \neq 0(p), p$ divides $1-j^{2 m}$, while for $j \equiv 0(p), p$ divides $j$ and so $p$ divides each $j\left(1-j^{2 m}\right), 3 \leqslant j \leqslant d$, and also $1-2^{2 m}$ and hence $p$ divides $b$. If $p-1$ does not divide $2 m$, then taking $j$ to be a primitive root for $p$, $j<p \leqslant d$, one has that $p$ does not divide $j\left(1-j^{2 m}\right)$, and so does not divide $b$.

Thus $b=g_{m}^{d} / h_{m}^{d}$ is the product of those odd primes $p$ with $p \leqslant d$ and $p-1$ dividing $2 m$. Since $h_{m}^{d}\left|s_{m}^{d}\right| g_{m}^{d}=b \cdot h_{m}^{d}, s_{m}^{d}=a \cdot h_{m}^{d}$ for some $a$ dividing $b$, giving the desired form for $a$.

For an odd prime $p$ with $p \leqslant d$ and $p-1$ dividing $2 m$, and either $2 m+1=p^{s}$ or $d<2 p$, one must have $p$ dividing $a$. For the case $2 m+1=p^{s}, s_{m}(\mathfrak{p})\left[M^{4 n}\right] \equiv 0$ $\bmod p$ for all manifolds and hence for all classes $\alpha$. For the case $d<2 p$, Proposition $5^{\prime}$ gives the divisibility.

Note. I am indebted to Gordon Keller for the argument using primitive roots in the above. For the following comments I am indebted to my son, Richard Stong.

Comment 1. If $p$ divides $h_{m}^{d}=\operatorname{gcd}\left\{\left(1-2^{2 m}\right), \ldots,\left(1-d^{2 m}\right)\right\}$, which is true for example if $p>d$ and $p-1$ divides $2 m$, and if $p<3 \times 10^{9}$ then

$$
\nu_{p}\left(h_{m}^{d}\right)= \begin{cases}1+\nu_{p}(m) & \text { for } d \geqslant 3 \text { or } d=2 \text { and } p \neq 1093,3511, \\ 2+\nu_{p}(m) & \text { for } d=2 \text { and } p=1093 \text { or } 3511 .\end{cases}
$$

Proof. If $p$ is an odd prime dividing $1-j^{2 m}$, then $p$ divides $1-j^{p-1}$ and so $1-j^{l}$ where $l=\operatorname{gcd}(p-1,2 m)$. Letting $j^{l}=x=1+s p^{r}, r>0$ and $s \neq 0 \bmod p$, 
one has

$$
\begin{aligned}
x^{p} & =1+\left\{s+\sum_{i=2}^{p}\left(\begin{array}{c}
p \\
i
\end{array}\right) s^{i} p^{(i-1) r-1}\right\} p^{r+1} \\
& =1+s^{\prime} p^{r+1} \quad \text { with } s^{\prime} \equiv s \bmod p
\end{aligned}
$$

and for $q \neq 0 \bmod p$,

$$
\begin{aligned}
x^{q} & =1+\left\{q s+\sum_{i=2}^{q}\left(\begin{array}{l}
q \\
i
\end{array}\right) s^{i} p^{(i-1) r}\right\} p^{r+1} \\
& =1+s^{\prime \prime} p^{r} \quad \text { with } s^{\prime \prime} \equiv q s \bmod p
\end{aligned}
$$

and so $x^{p^{j} q}=1+t p^{r+j}$ with $t \equiv q s \neq 0 \bmod p$. Thus $\nu_{p}\left(j^{p-1}-1\right)=\nu_{p}\left(j^{l}-1\right)$ and $\nu_{p}\left(j^{2 m}-1\right)=\nu_{p}\left(j^{l}-1\right)+\nu_{p}(2 m)$. From [5] one has $\nu_{p}\left(2^{p-1}-1\right)=1$ for $p<3 \times 10^{9}$ and $p \neq 1093,3511$, and in these exceptional cases $\nu_{p}\left(2^{p-1}-1\right)=2$ and $\nu_{p}\left(3^{p-1}-1\right)=1$.

Comment 2. The argument is also valid for $p>3 \times 10^{9}$ if $p^{2}$ does not divide $2^{2 m}-1$. In order that $p^{2}$ divide $2^{2 m}-1, m$ must be large, and in fact $m \geqslant 63$.

Proof. If $p^{2}$ divides $2^{2 m}-1=\left(2^{m}-1\right) \cdot\left(2^{m}+1\right)$, the two factors are relatively prime and so $p^{2}$ divides either $2^{m}-1$ or $2^{m}+1$. Thus $2^{m}+1 \geqslant p^{2}>9 \times 10^{18}$. Now $2^{63}>9 \times 10^{18}>2^{62}$ so one must have $m \geqslant 63$ at the minimum.

COMMENT 3. There are examples of primes $p$ dividing both $\left(1-2^{2 m}\right)$ and $\left(1-3^{2 m}\right)$ without having $(p-1) \mid 2 m$. Specifically, 2 and 3 are both quadratic residues of 73 , so 73 divides $1-2^{36}$ and $1-3^{36}$.

5. Edmonds' theorem. The arguments given by Edmonds actually prove considerably more, and this section will show how these arguments work.

Proposition 6. If $\phi: M^{n} \rightarrow N^{n}$ is a branched covering of closed oriented manifolds with oriented branch set $B_{\phi}$ and $H^{2}(N ; Q)=0$, then $\left[M^{n}\right]-(\operatorname{deg} \phi)\left[N^{n}\right] \in \operatorname{Tor}\left(\Omega_{*}\right)$.

Proof. If $B$ is oriented, then its covering $\phi^{-1} B_{\phi}$ is also orientable. Thus one has a factorization for $g$ and $\tilde{g}$,

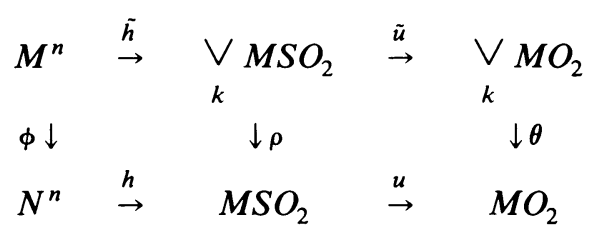

One now has $\tilde{u}^{*}\left(\mathfrak{p}_{1, k}\right)=U_{k}^{2}$ where $U_{k}$ is the Thom class in the $k$ th wedge summand of $V_{k} M S O_{2}$, and $\tilde{h}^{*}\left(U_{k}^{2}\right)=X\left(\tilde{\nu}_{k}\right) \tilde{h}^{*}\left(U_{k}\right)$ where $X\left(\tilde{\nu}_{k}\right)=c_{1}\left(\tilde{\nu}_{k}\right) \in H^{2}\left(\tilde{B}_{k} ; Z\right)$ is the Euler class or first Chern class of the normal bundle $\tilde{\nu}_{k}$ of $\tilde{B}_{k}$ in $M$. One then has

$$
\tilde{B}_{k} \stackrel{i}{\rightarrow} M^{n} \stackrel{\phi}{\rightarrow} N^{n} \stackrel{h}{\rightarrow} M S O_{2}
$$

with $(h \circ \phi \circ i)^{*}(U)=X\left(\tilde{\nu}_{k}^{k}\right)=c_{1}\left(\tilde{\nu}_{k}^{k}\right)=k c_{1}\left(\tilde{\nu}_{k}\right)$, where $\tilde{\nu}_{k}^{k}$ is the $k$ th tensor power of the complex line bundle $\tilde{\nu}_{k}$, and $U$ is the Thom class. Since $H^{2}(N, Q)=0$ one has 
$h^{*}(U)=0$ and so $k c_{1}\left(\tilde{\nu}_{k}\right)=0$ and $X\left(\tilde{\nu}_{k}\right)=0$ in $H^{2}\left(\tilde{B}_{k} ; Q\right)$. Thus $\mathfrak{p}_{1, k}=\tilde{g}^{*}\left(\mathfrak{p}_{1, k}\right)$ $=0$ in $H^{*}\left(M^{n} ; Q\right)$ and by Brand's formula $p\left(\tau(M)-\phi^{*} \tau(N)\right)=1$ in $H^{*}(M ; Q)$. Thus $\phi^{*} \mathfrak{p}(\tau(N))=\mathfrak{p}(\tau(M))$ rationally, so that $M$ and $(\operatorname{deg} \phi) N$ have the same Pontrjagin numbers.

Proposition 7. If $\phi: M^{n} \rightarrow N^{n}$ is a branched covering of closed oriented manifolds with $H^{4}(N ; Q)=0$, then $\left[M^{n}\right]-(\operatorname{deg} \phi)\left[N^{n}\right] \in \operatorname{Tor}\left(\Omega_{*}\right)$.

Proof. Two proofs will be given, with the first being a bit sophisticated.

Proof Number 1. Let $N \stackrel{f}{\rightarrow} B_{\operatorname{deg} \phi}$ be a classifying map for the branched covering $\phi$. According to Brand [4], $\left(B_{\mathrm{deg}} \phi\right) \otimes Q$ is a wedge of Eilenberg-Mac Lane spaces $K(Q, 4)$, and since $H^{4}(N, Q)=0$, the map $f$ in $\Omega_{*}\left(B_{\mathrm{deg}} \phi\right) \otimes Q$ lies in the image of $\Omega_{*}$ (point) $\otimes Q$. Thus some multiple of $\phi$ is cobordant to a trivial unbranched covering.

Proof Number 2. Essentially duplicating the argument of Proposition 6, one has $k^{2} \mathfrak{p}_{1}\left(\tilde{\nu}_{k}\right)=(g \circ \phi \circ i)^{*}\left(\mathfrak{p}_{1}\right)=0$ in $H^{*}(M ; Q)$ and so $\mathfrak{p}_{1, k}^{2}=\mathfrak{p}_{1}\left(\tilde{\nu}_{k}\right) \cdot \mathfrak{p}_{1, k}=0$ in $H^{*}(M, Q)$. Thus Brand's formula becomes

$$
\mathfrak{p}\left(\tau(M)-\phi^{*} \tau(N)\right)=1+\sum_{k}\left(1-k^{2}\right) \mathfrak{p}_{1, k} \in H^{*}(M ; Q) .
$$

For any partition $\omega$, one then has

$$
\begin{aligned}
& s_{\omega}(\mathfrak{p})(\tau(M))= s_{\omega}(\mathfrak{p})\left(\left(\tau(M)-\phi^{*} \tau(N)\right) \oplus \phi^{*} \tau(N)\right) \\
&= \sum_{\omega^{\prime} \cup \omega^{\prime \prime}=\omega} s_{\omega^{\prime}}\left(\mathfrak{p}\left(\tau(M)-\phi^{*} \tau(N)\right) \cup s_{\omega^{\prime \prime}}(\mathfrak{p})\left(\phi^{*} \tau(N)\right)\right) \\
&=\left\{\begin{array}{l}
\phi^{*}\left(s_{\omega}(\mathfrak{p})(\tau(N))\right) \text { if } \omega \neq\left(\omega^{\prime}, 1\right), \\
\phi^{*}\left(s_{\omega}(\mathfrak{p})(\tau(N))\right)+\phi^{*}\left(s_{\omega^{\prime}}(\mathfrak{p})(\tau(N))\right) \cdot\left(\sum_{k}\left(1-k^{2}\right) \mathfrak{p}_{1, k}\right)
\end{array}\right. \\
& \text { if } \omega=\left(\omega^{\prime}, 1\right),
\end{aligned}
$$

since $s_{\omega}(\mathfrak{p})\left(\tau(M)-\phi^{*} \tau(N)\right)$ is nonzero only for $\omega=(0)$ or (1), in rational cohomology. Thus $s_{\omega}(\mathfrak{p})[M]=(\operatorname{deg} \phi) s_{\omega}(\mathfrak{p})[N]$ except for $\omega=\left(\omega^{\prime}, 1\right)$, and

$$
\begin{aligned}
s_{\left(\omega^{\prime}, 1\right)}(\mathfrak{p})[M]-(\operatorname{deg} \phi) s_{\left(\omega^{\prime}, 1\right)}(\mathfrak{p}) & {[N]=\sum_{k}\left(1-k^{2}\right) \phi^{*}\left(s_{\omega^{\prime}}(\mathfrak{p})(\tau(N))\right) \mathfrak{p}_{1, k}[M] } \\
= & \sum_{k}\left(1-k^{2}\right) i^{*} \phi^{*}\left(s_{\omega^{\prime}}(\mathfrak{p})(\tau(N))\right)\left[\tilde{B}_{k} \cap \tilde{B}_{k}\right] \\
& =\sum\left(1-k^{2}\right)\left\langle s_{\omega^{\prime}}(\mathfrak{p})(\tau(N)),(\phi \circ i)_{*}\left[\tilde{B}_{k} \cap \tilde{B}_{k}\right]\right\rangle .
\end{aligned}
$$

However, $(\phi \circ i)_{*}\left[\tilde{B}_{k} \cap \tilde{B}_{k}\right] \in H_{n-4}\left(N^{n} ; Q\right) \cong H^{4}\left(N^{n} ; Q\right) \cong 0$ and so this number is zero. Thus $\left[M^{m}\right]-(\operatorname{deg} \phi)\left[N^{n}\right]$ has all Pontrjagin numbers zero.

Collecting together everything one knows, one then has

Proposition 8. Let $\phi: M^{n} \rightarrow S^{n}$ be a branched covering with $M^{n}$ closed and if $n=4$ assume $B_{\phi}$ orientable. Then $M^{n}$ is orientable and $\left[M^{n}\right] \in$ Tor $\Omega_{*}$. If $M^{n}$ is a Spin manifold or if $B_{\phi}$ is orientable, then $\left[M^{n}\right]=0$ in $\Omega_{*}$. 
Proof. $w_{1}(M)=\phi^{*} w_{1}\left(S^{n}\right)=0$, so $M$ is orientable. Let $\phi_{i}: M_{i} \rightarrow S^{n}$ be the restriction to the component $M_{i}$ of $M$ of $\phi$. Then $M_{i}$ with the orientation induced by the covering satisfies the conditions of Proposition 6 for $n=4$ and otherwise Proposition 7. Thus $\left[M_{i}\right]=\left[M_{i}\right]-\left(\operatorname{deg} \phi_{i}\right)\left[S^{n}\right] \in$ Tor $\Omega_{*}$. Reversing the orientation of $M_{i}$ does not change that, and hence the class of $M$ belongs to Tor $\Omega_{*}$ no matter how orientations are chosen.

If $M^{n}$ is a Spin manifold, one applies Corollary 3.5 of Bernstein and Edmonds [2], remarking as in $\$ 3$ that the hypothesis that $M$ have even Euler characteristic is unnecessary since $w_{n}\left(M^{n}\right)$ is the square of the Wu class $v_{n / 2}\left(M^{n}\right)$. This gives $\left[M^{n}\right]=0$ in $\Omega_{*}$. If $B_{\phi}$ is orientable, one applies the corollary from $\S 3$ to obtain $\left[M^{n}\right]=0$ in $\mathfrak{R}$. Finally, one recalls the Tor $\Omega_{*}$ injects into $\mathfrak{N}_{*}$, and hence $M^{n}$ is an oriented boundary in both of these cases.

Note. For $n=4, \pi: C P^{2} \rightarrow C P^{2} /$ conjugation $=S^{4}$ has nonorientable branch set. One also has the $k$-dric $\rho: Q_{k}^{4} \rightarrow \mathbf{C} P^{2}$ branched along the $k$-dric $Q_{k}^{2}=\left\{z \in \mathbf{C} P^{2} \mid z_{0}^{k}\right.$ $\left.+z_{1}^{k}+z_{2}^{k}=0\right\}$ with $Q_{k}^{4}$ a Spin manifold for $k$ even and $Q_{k}^{2}$ does not meet $R P^{2}$. Thus the composite $\rho \circ \pi: Q_{k}^{4} \rightarrow S^{4}$ is a branched cover with $Q_{k}^{4}$ being Spin, provided $k$ is even and $\mathfrak{p}_{1}\left[Q_{k}^{4}\right]=\left(4-k^{2}\right) k \neq 0$ for $k=4$.

To see that there is a branched covering $\phi: P^{5} \rightarrow S^{5}$ with $\left[P^{5}\right] \neq 0$, one may proceed as follows. One has a cofibration $R P^{\infty} \rightarrow B_{2} \underset{\sim}{\rightarrow} M\left(\mu_{2}\left(\gamma_{2}\right)\right)$ where $B_{2}$ is Brand's classifying space for 2-fold branched covers, and $\gamma_{2}$ is the 2-plane bundle over $B O_{2}$, and a cofibration $M\left(\mu_{2}\left(\gamma_{2}\right) \mid S \gamma_{2}\right) \rightarrow M\left(\mu_{2}\left(\gamma_{2}\right)\right) \stackrel{\beta}{\rightarrow} M\left(\mu_{2}\left(\gamma_{2}\right) \oplus \gamma_{2}\right)$. Now $M\left(\mu_{2}\left(\gamma_{2}\right) \mid S \gamma_{2}\right)=M\left(\mu_{2}\left(\gamma_{1} \oplus 1\right)\right) \cong M\left(\mu_{1} \oplus 1\right) \cong \Sigma R P^{\infty}$. The composite $\beta \circ \alpha: B_{2}$ $\rightarrow M\left(\mu_{2}\left(\gamma_{2}\right) \oplus \gamma_{2}\right)$ is then a homotopy equivalence. It is readily seen to induce an isomorphism on unoriented bordism, hence on $Z_{2}$ homology, while $\alpha$ and $\beta$ are isomorphisms in $Z_{p}$ homology for odd $p$. Finally, both spaces are simply connected. One then observes that $\mu\left(\gamma_{2}\right) \oplus \gamma_{2}$ is orientable to produce a map $\theta: M\left(\mu_{2}\left(\gamma_{2}\right) \oplus \gamma_{2}\right)$ $\rightarrow K(Z ; 4) \times K\left(Z_{2}, 5\right)$ via the classes $\phi(1)$ and $\phi\left(w_{1}\right)$, where $\phi$ is the Thom isomorphism. $\theta$ induces an isomorphism in mod 2 cohomology through dimension 7 . Thus $\pi_{5}\left(B_{2}\right) \cong Z_{2}$ (plus possible odd torsion) with nontrivial image in $H_{5}\left(B_{2} ; Z_{2}\right)$. The branch set for this map is the nonzero class in $\mathfrak{R}_{1}\left(B O_{2}\right)$ with $w_{1}$ being the nonzero number.

6. Coverings of spheres. Since all classes of manifolds branched over $S^{n}$ with $n>5$ belong to Tor $\Omega_{n} \subset \mathfrak{R}_{n}$, one should analyze the possible Stiefel-Whitney numbers for manifolds branched over $S^{n}$. This section will do so.

OBSERVATION. The set $B\left(S^{n}\right)$ of classes $\left[M^{n}\right] \in \Omega_{n}$, with $\phi: M^{n} \rightarrow S^{n}$ a branched covering, is a subgroup of $\Omega_{n}$.

Proof. If $\phi: M^{n} \rightarrow S^{n}$ is a branched covering, $B_{\phi} \subset S^{n}$ is a proper closed subset of $S^{n}$ and hence one may find a closed disc contained in $S^{n}-B_{\phi}$. By reparametrizing $S^{n}$, one may assume that disc is the "southern" hemisphere $D_{-}^{n}$ and hence that $B_{\phi} \subset$ interior $\left(D_{+}^{n}\right)$. If $\psi: N^{n} \rightarrow S^{n}$ is a second branched covering one may similarly suppose $B_{\psi} \subset$ interior $\left(D_{-}^{n}\right)$. The union $\phi \cup \psi: M^{n} \cup N^{n} \rightarrow S^{n}$ is then a branched cover and gives the sum of the classes in $B\left(S^{n}\right)$.

Note. If $\phi: M^{n} \rightarrow S^{n}$ and $\psi: N^{n} \rightarrow S^{n}$ both have degree $d$, one may realize the sum by a branching of degree $d$. One simply joins $\phi^{-1}\left(D_{+}^{n}\right)$ and $\psi^{-1}\left(D_{-}^{n}\right)$ along their 
common boundaries which are copies of $S^{n-1} \times\{1,2, \ldots, d\}$. The resulting manifold is obtained by surgery on $d$ copies of $D^{n} \times S^{0}$ in $M \cup N$. This phenomenon is much more general since one could sew together two $d$-fold coverings over $M^{\prime n}$ and $N^{\prime n}$ to obtain a $d$-fold covering of $M^{\prime n} \# N^{\prime n}$.

If one now considers a branched covering $\phi: M^{n} \rightarrow S^{n}$ with $n>5$, one has by Brand's formula

$$
\begin{aligned}
w\left(M^{n}\right) & =\phi^{*} w\left(S^{n}\right)\left\{1+U_{e v}+w_{1} U_{e v}+\cdots+w_{1}^{t} U_{e v}+\cdots\right\} \\
& =\left\{1+U_{e v}+w_{1} U_{e v}+\cdots+w_{1}^{t} U_{e v}+\cdots\right\},
\end{aligned}
$$

where $U_{e v}=\Sigma_{k \text { even }} U_{k}$ and induced homomorphisms are ignored. Letting $B_{e v}$ be the points of $\phi^{-1}\left(B_{\phi}\right)$ of even local branching degree, $B_{e v}$ is the submanifold of $M^{n}$ dual to the class $U_{e v}$. One then has

$$
w\left(B_{e v}\right)=\frac{1}{1+w_{1}}=1+w_{1}+w_{1}^{2}+w_{1}^{3}+\cdots+w_{1}^{n-2}
$$

and

$$
w\left(\tilde{\nu}_{e v}\right)=1+w_{1}+w_{2}
$$

where $w_{2}$ is the restriction of $U_{e v}$ to $B_{e v}$.

Lemma 3. The Stiefel-Whitney numbers of $M^{n}$ are given by

$$
w_{i_{1}} \cdots w_{i_{r}}\left[M^{n}\right]= \begin{cases}0 & \text { if any } i_{\alpha}=1 \\ w_{1}^{n-r} w_{2}^{r-1}\left[B_{e v}^{n-2}\right] & \text { if each } i_{\alpha}>1\end{cases}
$$

Proof. Since $w_{1}(M)=0$, the first formula is obvious. For the second, one has from the proof of Proposition $2^{\prime}$ that $w_{\omega}\left[M^{n}\right]=\hat{w}_{\omega}\left[R P\left(\tilde{\nu}_{e v} \oplus 1\right)\right]$, where

$$
\hat{w}\left(R P\left(\tilde{\nu}_{e v} \oplus 1\right)\right)=1+U+w_{1} U+\cdots+w_{1}^{t} U+\cdots
$$

and $U=c^{2}+w_{1} c+w_{2}$ with $c U=0$, and here the classes $w_{1}^{t} U$ are actual products. Thus

$$
\begin{aligned}
\hat{w}_{i_{1}} \cdots \hat{w}_{i_{r}}\left[R P\left(\tilde{\nu}_{e v} \oplus 1\right)\right] & =w_{1}^{i_{1}+\cdots+i_{r}-2 r} U^{r}\left[R P\left(\tilde{\nu}_{e v} \oplus 1\right)\right] \\
& =w_{1}^{n-2 r} w_{2}^{r-1} U\left[R P\left(\tilde{\nu}_{e v} \oplus 1\right)\right]=w_{1}^{n-2 r} w_{2}^{r-1}\left[B_{e v}\right] .
\end{aligned}
$$

Note. One may obtain the second formula directly by considering the map $g$ : $M \rightarrow M\left(\tilde{\nu}_{e v}\right)$ by collapsing. Then $w_{i_{1}} \cdots w_{i_{r}}[M]=g^{*}\left(\Phi\left(w_{1}^{i_{1}-2}\right) \cdots \Phi\left(w_{1}^{i_{r}-2}\right)\right)[M]=$ $g^{*}\left(\Phi\left(w_{1}^{n-2 r} w_{2}^{r-1}\right)\right)[M]$, where $\Phi$ is the Thom isomorphism. This is $\left\langle\Phi\left(w_{1}^{n-2 r} w_{2}^{r-1}\right)\right.$, $\left.g_{*}[M]\right\rangle=\left\langle w_{1}^{n-2 r} w_{2}^{r-1}, \phi g_{*}[M]\right\rangle$ where $\phi$ is the homology Thom isomorphism, and $\phi g_{*}[M]=\left[B_{e v}\right]$ gives the result.

Note. This argument does not depend on the use of $S^{n}$, and shows that $x\left(w_{1}^{a} U_{e v}\right)[M]=x w_{1}^{a}\left[B_{e v}\right]$ for any branching and class $x$, i.e. $w_{1}^{a} U$ acts like a product.

Note. This gives an alternative proof that $\phi: M^{n} \rightarrow S^{n}$ with $M$ Spin implies $M$ bounds without using a category argument. Use $w_{n}=v_{n / 2}^{2}$ to get an equivalent number $w_{n}[M]=\sum w_{i_{1}} \cdots w_{i_{r}}[M]$ with $r>0$. Then $w_{i_{1}} \cdots w_{i_{r}}[M]=$ $w_{n-2 r+2} w_{2}^{r-1}[M]=0$ whenever $r>0$, so all numbers of $M$ are zero. 
LEMMA 4. The $W u$ class of $B_{e v}$ is given by

$$
v=1+w_{1}+w_{1}^{3}+\cdots+w_{1}^{2^{t}-1}+\cdots,
$$

and

$$
0=\bar{w}_{n-2}\left(\tilde{\nu}_{e v}\right)\left[B_{e v}\right]=\sum_{k=0}^{[(n-2) / 2]}\left(\begin{array}{c}
n-2-k \\
k
\end{array}\right) w_{1}^{n-2-2 k} w_{2}^{k}\left[B_{e v}\right]
$$

Proof. The first formula is obtained by $v=\mathrm{Sq}^{-1} w$ with $w=1+w_{1}+w_{1}^{2}$ $+\cdots+w_{1}^{t}+\cdots$. One calculates the dual Stiefel-Whitney class $\bar{w}=1 / w$ of $M$ by calculation in $\mathrm{MO}_{2}$, and to do that calculation one may calculate in $\mathrm{BO}_{2}$, following Brand. One has

$$
\frac{1+w_{1}}{1+w_{1}+w_{2}}=\frac{1+w_{1}+w_{2}+w_{2}}{1+w_{1}+w_{2}}=1+\frac{w_{2}}{1+w_{1}+w_{2}},
$$

and so

$$
\bar{w}(M)=1+U_{e v} /\left(1+w_{1}+U_{e v}\right)
$$

expanded in the usual formal way. Thus

$$
0=\bar{w}_{n}\left[M^{n}\right]=\left(\frac{1}{1+w_{1}+w_{2}}\right)\left[B_{e v}\right]=\bar{w}_{n-2}\left(\tilde{\nu}_{e v}\right)\left[B_{e v}\right] .
$$

Finally, the degree $i$ component of $1 /\left(1+w_{1}+w_{2}\right)$ is $\sum_{j=0}^{[i / 2]}\left({ }_{j}^{i-2 j+j}\right) w_{1}^{i-2 j} w_{2}^{j}$, which is easily seen by induction on $i$.

Note. The condition $\bar{w}_{n-2}\left(\tilde{\nu}_{e v}\right)\left[B_{e v}\right]=0$ is equivalent to the assertion that $R P\left(\tilde{\nu}_{e v}\right) \stackrel{\phi \times c}{\rightarrow} S^{n} \times R P^{\infty}$ bounds. One has $w\left(R P\left(\tilde{\nu}_{e v}\right)\right)=1$ and the only relation is $0=c^{n-1}\left[R P\left(\tilde{\nu}_{e v}\right)\right]$ which is this relation on $B_{e v}$.

LEMMA 5. If $n$ is even, $n>4$, then $M^{n}$ bounds.

Proof. One considers the numbers $w_{1}^{a} w_{2}^{b}\left[B_{e v}\right]$ with $a+2 b=n-2$. Thus $a$ must be even.

For $b$ odd and $a>0$, one has

$$
\begin{aligned}
w_{1}^{2 p+2} w_{2}^{2 q+1}\left[B_{e v}\right] & =w_{1}\left\{\left(w_{1} w_{2}\right) \cdot\left(w_{1}^{p} w_{2}^{q}\right)^{2}\right\}\left[B_{e v}\right] \\
& =\operatorname{Sq}^{1}\left\{\left(\mathrm{Sq}^{1} w_{2}\right) \cdot x^{2}\right\}\left[B_{e v}\right]=0
\end{aligned}
$$

for $\mathrm{Sq}^{1} \mathrm{Sq}^{1}=0$ and $\mathrm{Sq}^{1}\left(x^{2}\right)=0$.

For $b>0$ and even, one has

$$
\begin{aligned}
w_{1}^{2 p} w_{2}^{2 q}\left[B_{e v}\right] & =\operatorname{Sq}^{(n-2) / 2}\left(w_{1}^{p} w_{2}^{q}\right)\left[B_{e v}\right] \\
& = \begin{cases}0 & \text { if }(n-2) / 2 \neq 2^{t}-1, \\
w_{1}^{2^{t}-1+p_{p}} w_{2}^{q}\left[B_{e v}\right] & \text { if }(n-2) / 2=2^{t}-1,\end{cases}
\end{aligned}
$$

by the formula for $v$. One notes that $2^{t}-1+p>0$ and that the power of 2 dividing $b$ has been reduced in the second case. Inductively, on this power of 2 , these numbers are zero. 
The only possible nonzero numbers are then those with $a=0$ and $b$ odd or with $b=0$, with the latter only for $(n-2) / 2=2^{t}-1$ by the same Wu class argument.

Now

$$
w_{2}^{2 q+1}\left[B_{e v}\right]=w_{2}^{2 q+2}\left[M^{n}\right]=\left(w_{2}^{2}\right)^{q+1}\left[M^{n}\right] \equiv\left(\mathfrak{p}_{1}\right)^{q+1}\left[M^{n}\right] \bmod 2
$$

and since $\left[M^{n}\right] \in \operatorname{Tor}\left(\Omega_{n}\right)$, this is zero. (Note. This does not hold for $n=4$, and is the crucial number for the case.)

If $(n-2) / 2=2^{t}-1, n=2^{t+1}$, and the coefficient of $w_{1}^{2^{t+1}-2}$ in $\bar{w}_{n-2}\left(\tilde{v}_{e v}\right)$ is 1 . Since all other $w_{1}^{a} w_{2}^{b}\left[B_{e v}\right]$ are zero, one must have $w_{1}^{2^{t+1}-2}\left[B_{e v}\right]=0$.

The situation for $n$ odd is much harder. Since $\Omega_{1}=\Omega_{3}=\Omega_{7}=0$ and since $\Omega_{5} \cong Z_{2}$ has a nonzero class known to branch over $S^{5}$, one may suppose $n \geqslant 9$. One may then divide up into the cases $2^{k+1}>n>2^{k}$, where with no loss $k \geqslant 3$. (Everything could be checked for smaller $k$.) Further, it is convenient to consider

$$
2^{k}+2^{r+1}-3 \geqslant n>2^{k}+2^{r}-3,
$$

with $1 \leqslant r \leqslant k$. (Note. For $r=1, n=2^{k}+1$ only, and for $r=k, n=2^{k+1}-1$ only.)

LEMMA 6. For $n$ odd, $w_{1}^{n-2 p} w_{2}^{p^{-1}}\left[B_{e v}\right]=0$ if $p$ is odd.

Proof. $w_{1}^{n-2 p} w_{2}^{p-1}\left[B_{e v}\right]=\operatorname{Sq}^{1}\left(w_{1}^{(n-2 p-1) / 2} w_{2}^{(p-1) / 2}\right)^{2}\left[B_{e v}\right]=0$ for $\operatorname{Sq}^{1} x^{2}=0$.

LEMMA 7. For $n$ odd, $2^{k+1}>n>2^{k}, w_{1}^{n-2 p} w_{2}^{p-1}\left[B_{e v}\right]=0$ except for $((n-1) / 4)$ $-\left(2^{k-2}-1\right) \leqslant p / 2 \leqslant((n-1) / 4)$.

Proof. To have $w_{1}^{n-2 p} w_{2}^{p-1}\left[B_{e v}\right] \neq 0$ one must have $2(p-1) \leqslant n-2$ and since $n$ is odd, $2(p-1) \leqslant n-3$ or $p / 2 \leqslant((n-1) / 4)$. From $2^{k+1}>n>2^{k}$, one has $2^{k}-1>(n-2) / 2>2^{k-1}-1$. Since $v=1+w_{1}+w_{1}^{3}+\cdots+w_{1}^{2^{t}-1}+\cdots$ and $v_{i}=0$ if $i>[(n-2) / 2]$, one has $v=1+w_{1}+w_{1}^{3}+\cdots+w_{1}^{2^{k-1}-1}$ and $w_{1}^{2^{k}-1}$ $=0$. To have $w_{1}^{n-2 p} w_{2}^{p-1}\left[B_{e v}\right] \neq 0$, one must then have $n-2 p<2^{k}-1$ or $\left(n+1-2^{k}\right) / 2<p$ and $\left(n+1-2^{k}\right) / 2+1 \leqslant p$. Thus $(n-1) / 2-\left(2^{k-1}-2\right) \leqslant$ $p$ and dividing by 2 gives the result.

LEMMA 8. For $n$ odd, the numbers $w_{1}^{n-2 p} w_{2}^{p^{-1}}\left[B_{e v}\right]$ depend only on the numbers $w_{1}^{n-2^{t+1}} w_{2}^{2^{t}-1}\left[B_{e v}\right]$ with $2^{t} \leqslant p$.

Proof. If $p \neq 2^{t}$, there are integers $a, b$ with $a+b=p-1$ and $\left(\begin{array}{c}a \\ b\end{array}\right) \equiv 1 \bmod 2$, for example, if $p=2^{r}(2 s+1), r, s>0$, one may let $b=2^{r}$. One then has

$$
0=\mathrm{Sq}^{2 b}\left(w_{1}^{n-2 p} w_{2}^{a}\right)\left[B_{e v}\right]
$$

and

$$
\operatorname{Sq}\left(w_{1}^{n-2 p} w_{2}^{a}\right)=w_{1}^{n-2 p} w_{2}^{a}\left(1+w_{1}\right)^{n-2 p}\left(1+w_{1}+w_{2}\right)^{a} .
$$

One wishes to examine terms of $\left(1+w_{1}\right)^{n-2 p}\left(1+w_{1}+w_{2}\right)^{a}$ of dimension $2 b$, but

$$
\left(1+w_{1}\right)^{n-2 p}\left(1+w_{1}+w_{2}\right)^{a}=\sum_{i=0}^{a}\left(\begin{array}{c}
a \\
i
\end{array}\right) w_{2}^{i}\left(1+w_{1}\right)^{n-2 p+a-i},
$$


so that the coefficient of $w_{2}^{b}$ is $\left(\begin{array}{c}a \\ b\end{array}\right)$. Thus one has an equation

$$
w_{1}^{n-2 p} w_{2}^{p-1}\left[B_{e v}\right]=w_{1}^{n-2 p} w_{2}^{a} \cdot w_{2}^{b}\left[B_{e v}\right]=\sum_{q<p} \alpha_{q} w_{1}^{n-2 q} w_{2}^{q-1}\left[B_{e v}\right]
$$

where $\alpha_{q}$ depends only on $n, p$, and $a$, and of course $q$. Inductively, the result holds for $q<p$, giving the result.

COROLlaRY. For $n$ odd, $2^{k}+2^{r+1}-3 \geqslant n>2^{k}+2^{r}-3,2^{k+1}>n$, $\operatorname{dim}_{Z_{2}}\left\{B\left(S^{n}\right)\right\} \leqslant k-r$.

Proof. From Lemmas 6 and 7, the class of $M^{n}$ is determined by the numbers $w_{1}^{n-2 p} w_{2}^{p-1}\left[B_{e v}\right]$ with $p=2^{s+1}$ and

$$
((n-1) / 4)-\left(2^{k-2}-1\right) \leqslant 2^{s} \leqslant((n-1) / 4) .
$$

Now $((n-1) / 4)-\left(2^{k-2}-1\right)>\left(2^{k}+2^{r}-4\right) / 4-\left(2^{k-2}-1\right)=2^{r-2}$, so $s \geqslant r-$ 1. Also $n-1<2^{k+1}$, and so $s<k-1$. Thus $r-1 \leqslant s \leqslant k-2$. Thus one has $k-r$ choices for $s$.

COROLlARY. If $n=2^{k+1}-1, M^{n}$ bounds.

Proof. This is the case $r=k$, and $B\left(S^{n}\right)=0$.

LEMMA $8^{\prime}$. If $n$ is odd and $w_{1}^{n-2 p} w_{2}^{p-1}\left[B_{e v}\right]=0$ for $p<2^{s+1}$, then $w_{1}^{n-2 p} w_{2}^{p-1}\left[B_{e v}\right]$ $=0$ for $2^{s+1}<p<2^{s+1}+2^{s}$.

Proof. This requires more precision in the proof of Lemma 8. Assume $w_{1}^{n-2 p^{\prime}} w_{2}^{p^{\prime-1}}\left[B_{e v}\right]=0$ for $2^{s+1}<p^{\prime}<p$, which is true for $p^{\prime}=2^{s+1}+1$ since $p^{\prime}$ is then odd. One then has the formula

$$
w_{1}^{n-2 p} w_{2}^{p-1}\left[B_{e v}\right]=\alpha_{2^{s+1}} w_{1}^{2^{s+2}-1} w_{2}^{2^{s+1}-1}\left[B_{e v}\right]
$$

since all other terms are zero. The coefficient of $w_{2}^{2^{s+1}-1-a}$ in

$$
\left(1+w_{1}\right)^{n-2 p}\left(1+w_{1}+w_{2}\right)^{a}
$$

is

$$
\left(\begin{array}{c}
a \\
2^{s+1}-1-a
\end{array}\right)\left(1+w_{1}\right)^{n-2 p+a-\left(2^{s+1}-1-a\right)}
$$

and the binomial coefficient can be nonzero only when $2^{s+1}-1-a=0$. If one can choose $a \neq 2^{s+1}-1$ one then has $\alpha_{2^{s+1}}=0$ and so $w_{1}^{n-2 p} w_{2}^{p-1}\left[B_{e v}\right]=0$. For $p=2^{s+1}+t, t<2^{s}$, one may let $b=2^{s}, a=2^{s}+t-1$ to obtain $a<2^{s+1}-1$.

LEMMA 9. If $n \equiv\left(2^{q}-1\right) \bmod 2^{q+1}$ and $w_{1}^{n-2 p} w_{2}^{p-1}\left[B_{e v}\right]$ is zero for $p<2^{s}$, nonzero for $p=2^{s}$ and $s>q$, then $n<2^{s+1}+2^{q}$.

Proof. Suppose $n-2^{s+1}-2^{q} \geqslant 0$, and consider

$$
0=v_{2^{q}}\left(w_{1}^{n-2^{s+1}-2^{q}} w_{2}^{2^{s-1}}\right)\left[B_{e v}\right]=\operatorname{Sq}^{2^{q}}\left(w_{1}^{n-2^{s+1}-2^{q}} w_{2}^{2^{s-1}}\right)\left[B_{e v}\right] .
$$


One has

$$
\begin{aligned}
& \operatorname{Sq}\left(w_{1}^{n-2^{s+1}-2^{q}} w_{2}^{2^{s}-1}\right) \\
& =w_{1}^{n-2^{s+1}-2^{q}} w_{2}^{2^{s}-1}\left(\sum_{j=0}^{2^{s}-1}\left(\begin{array}{c}
2^{s}-1 \\
j
\end{array}\right) w_{2}^{j}\left(1+w_{1}\right)^{n-2^{s+1}-2^{q}+2^{s}-1-j}\right)
\end{aligned}
$$

and in the terms for $\mathrm{Sq}^{2^{q}}$ the powers $w_{2}^{j}$ occur for $0 \leqslant j \leqslant 2^{q-1}<2^{s-1}$. By Lemma $8^{\prime}$ only the term with $j=0$ can be nonzero, giving

$$
0=\left(\begin{array}{c}
n-2^{s+1}-2^{q}+2^{s}-1 \\
2^{q}
\end{array}\right) w_{1}^{n-2^{s+1}} w_{2}^{2^{s-1}}\left[B_{e v}\right] .
$$

Now $n-2^{s+1}-2^{q}+2^{s}-1=n-2^{s}-2^{q}-1 \geqslant 2^{s}-1>0$ is congruent to -2 $\bmod 2^{q+1}$, and the binomial coefficient is $1 \bmod 2$. Thus one has a contradiction, and so $n<2^{s+1}+2^{q}$.

LEMMA 10. If $n \equiv\left(2^{q}-1\right) \bmod 2^{q+1}$, then $B\left(S^{n}\right)=0$ except for $n=2^{k}+\left(2^{q}-1\right)$, $k>q$, and for $n=2^{k}+\left(2^{q}-1\right)$ with $k>q$,

$$
\operatorname{dim}_{Z_{2}} B\left(S^{n}\right) \leqslant \begin{cases}1, & k=q+1 \\ 2, & k>q+1\end{cases}
$$

Proof. If $n=2^{q}-1, B\left(S^{n}\right)=0$ by the second corollary to Lemma 8 . Thus, one may suppose $n=2^{q+1} t+\left(2^{q}-1\right)$ with $t>0$.

If $t=1$, one has $2^{q+2}>n>2^{q+1}$ and $2^{q+1}+2^{q+1}-3>n>2^{q+1}+2^{q}-3$, i.e. $k=q+1, r=q$. By the first corollary to Lemma $8, \operatorname{dim}_{Z_{2}} B\left(S^{n}\right) \leqslant k-r=(q+$ 1) $-q \leqslant 1$.

Now suppose $t>1$, and choose $k, r$ with $2^{k+1}>n>2^{k}, 2^{k}+2^{r+1}-3 \geqslant n>2^{k}$ $+2^{r}-3$. Because $t>1, k \geqslant q+2$, and $n \neq 2^{k+1}-1$ so $k>r$. For $r<q$, the interval $\left(2^{k}+2^{r}-3,2^{k}+2^{r+1}-3\right]$ contains no integer congruent to $2^{q}-1$ $\bmod 2^{q+1}$, and hence $r \geqslant q$.

If $r>q$, then from the argument for the first corollary of Lemma 8, $w_{1}^{n-2^{s+1}} w_{2}^{2^{s}-1}\left[B_{e v}\right] \neq 0$ only for $r \leqslant s \leqslant k-1$, and let $s^{\prime}$ be the smallest such $s$ giving a nonzero value, so that $s^{\prime} \geqslant r>q$. By Lemma $9, n<2^{s^{\prime}+1}+2^{q} \leqslant 2^{k}+2^{q}$, so $2^{k}+2^{r}-1 \leqslant n \leqslant 2^{k}+2^{q}-1$ contradicting the assumption $r>q$, or that $s^{\prime}$ exists. Thus $B\left(S^{n}\right)=0$.

For $r=q, 2^{k}+2^{q+1}-3 \geqslant n>2^{k}+2^{q}-3$ gives $n=2^{k}+\left(2^{q}-1\right)$. Consider the subspace of $B\left(S^{n}\right)$ consisting of those manifolds for which $w_{1}^{n-2^{q+1}}\left[B_{e v}\right]=0$. On this subspace one has $w_{1}^{n-2^{s+1}} w_{2}^{2^{s}-1}\left[B_{e v}\right] \neq 0$ only for $q+1 \leqslant s \leqslant k-1$, and letting $s^{\prime}$ be the smallest such $s$, one has $s^{\prime}>q$. By Lemma $9, n<2^{s^{\prime}+1}+2^{q}$ and so $s^{\prime}+1=k$, i.e. $s^{\prime}=k-1$. Thus, the subspace of $B\left(S^{n}\right)$ for which $w_{1}^{n-2^{q+1}} w_{2}^{2^{q}-1}\left[B_{e v}\right]=0$ has dimension at most one and is detected by $w_{1}^{n-2^{k}} w_{2}^{2^{k-1}-1}\left[B_{e v}\right]$. Hence, $\operatorname{dim}_{Z_{2}} B\left(S^{n}\right) \leqslant 2$. 
Combining all of the pieces, one has

Proposition 9'. For $n$ even, $B\left(S^{n}\right)=\Omega_{n} \cong Z$ if $n=0$ or 4 , and $B\left(S^{n}\right)=0$ otherwise. For $n$ odd, $n \equiv\left(2^{q}-1\right) \bmod 2^{q+1}, B\left(S^{n}\right)=0$ except possibly for $n=2^{k}+$ $\left(2^{q}-1\right), k>q$, and for $n=2^{k}+\left(2^{q}-1\right)$ one has

$$
\operatorname{dim}_{Z_{2}} B\left(S^{n}\right) \leqslant \begin{cases}1, & \text { if } k=q+1 \\ 2, & \text { if } k>q+1 .\end{cases}
$$

Notes. (1) The arguments actually work for manifolds $S^{n}$ more general than the sphere. One could assume $w\left(S^{n}\right)=1$ and take $B\left(S^{n}\right)$ to be the subgroup of $\Omega_{n}$ (or $\left.\mathfrak{N}_{n}\right)$ generated by the classes $\left[M^{n}\right]-(\operatorname{deg} \phi)\left[S^{n}\right]$ (i.e. use $\phi: M^{n} \rightarrow S^{n}$ a union of branched coverings). In the proof of Lemma 5 one has $w_{2}^{2 q+1}\left[B_{e v}\right]=\bar{w}_{n-2}\left(\tilde{\nu}_{e v}\right)\left[B_{e v}\right]$ $=0$ if $n \neq 2^{t+1}$, while $n=2^{t+1}$ gives only $w_{1}^{2^{t+1}-2}\left[B_{e v}\right]=w_{2}^{2^{t}-1}\left[B_{e v}\right]$. Assuming $H^{4}\left(S^{n} ; Q\right)=0$, one has the full result for the classes $\left[M^{n}\right]-(\operatorname{deg} \phi)\left[S^{n}\right]$, and if $\left[S^{n}\right]=0$ in $\Omega_{n}$ the full result for the classes $\left[M^{m}\right]$. With no extra assumption one has an unoriented result with an extra case $n=2^{t+1}$, with $\operatorname{dim}_{Z_{2}}\left(B\left(S^{n}\right)\right) \leqslant 1$.

(2) For $n=2^{k}+2^{q}-1, k>q$, consider the Dold manifold

$$
P^{n}=P\left(2^{q}-1,2^{k-1}\right)=S^{2^{q}-1} \times \mathbf{C} P^{2^{k-1}} /(-1 \times \text { conjugation })
$$

and the Milnor hypersurface

$$
H^{n}=H\left(2^{q}, 2^{k}\right)=\left\{([x],[y]) \in R P^{2^{q}} \times R P^{2^{k}} \mid \sum_{i=0}^{2^{q}} x_{i} y_{i}=0\right\} .
$$

Over each of these manifolds one has a 2-plane bundle $\eta$,

$$
S^{2^{q}-1} \times(\text { Hopf bundle }) /(-1 \times \text { conjugation })
$$

or $\xi_{1} \oplus \xi_{2} \mid H^{n}$ respectively, and hence a composite map $M^{n} \stackrel{\eta}{\rightarrow} B \mathrm{O}_{2} \stackrel{i}{\rightarrow} M \mathrm{O}_{2}$. One has $w\left(P^{n}\right)=(1+c)^{2^{q}-1}(1+c+d)^{2^{k-1}+1}=\left(1+d+c d+\cdots+c^{2^{q}-1} d\right)\left(1+d^{2^{k-1}}\right)$ and

$$
w\left(H^{n}\right)=\frac{(1+\alpha)^{2^{q}+1}(1+\beta)^{2^{k}+1}}{(1+\alpha+\beta)}=\left(1+\frac{\alpha \beta}{1+\alpha+\beta}\right)\left(1+\alpha^{2^{q}}+\beta^{2^{k}}\right)
$$

with the classes $d^{2^{k-1}}, \alpha^{2^{q}}$, and $\beta^{2^{k}}$ making no contributions to Stiefel-Whitney numbers. Thus, the elements $\left(M^{n}, i \circ \eta\right)$ in $\mathfrak{N}_{n}\left(M O_{2}\right)$ have the same characteristic numbers as if $w\left(M^{n}\right)$ were $(i \circ \eta)^{*}\left(1+\sum w_{1}^{t} U_{2}\right)$. Making the maps $i \circ \eta$ transverse to $\mathrm{BO}_{2} \subset M \mathrm{O}_{2}$, one obtains codimension 2 submanifolds $B_{e v}\left(M^{n}\right)$, and this can be done explicitly to give

$$
B_{e v}(P)=P\left(2^{q}-1,2^{k-1}-1\right) \quad \text { and } \quad B_{e v}(H)=H\left(2^{q}-1,2^{k}-1\right)
$$

with

$$
\begin{aligned}
& w\left(B_{e v}(P)\right)=1 /(1+c), w\left(\tilde{\nu}_{e v}\right)=1+c+d, \\
& w\left(B_{e v}(H)\right)=1 /(1+\alpha+\beta), w\left(\tilde{\nu}_{e v}\right)=1+(\alpha+\beta)+\alpha \beta .
\end{aligned}
$$

One then has

$$
w_{1}^{n-2^{q+1}} w_{2}^{2^{q}-1}\left[B_{e v}(P)\right]=0 \quad(\text { if } k>q+1), \quad w_{1}^{n-2^{k}} w_{2}^{2^{k-1}-1}\left[B_{e v}(P)\right] \neq 0,
$$


and

$$
w_{1}^{n-2^{q+1}} w_{2}^{2^{q}-1}\left[B_{e v}(H)\right] \neq 0, \quad w_{1}^{n-2^{k}} w_{2}^{2^{k-1}-1}\left[B_{e v}(H)\right]=0 \quad(\text { if } k>q+1) .
$$

These provide examples of manifolds with the correct Stiefel-Whitney numbers, and with $B_{e v}(M)$ actually having the correct Stiefel-Whitney class structure, to all of the exceptional cases in Proposition 9'. Thus the argument cannot be improved at the Stiefel-Whitney number level.

In the case $n=5$, there are maps $f: M^{5} \rightarrow M O_{2}$, not lifting to $B O_{2}$, for which Brand's stable bundle actually pulls back to $\tau\left(M^{5}\right)$. For $P^{5}$, one has the branching $\phi: P^{5} \rightarrow S^{1} \times S^{4}=P(1,2) /(1 \times$ conjugation $)$, and for $H^{5}$ one has the involution $T\left([x],\left[y_{0}, y_{1}, y_{2}, y_{3}, y_{4}\right]\right)=\left([x],\left[y_{0}, y_{1}, y_{2},-y_{3},-y_{4}\right]\right)$ giving a branched cover $H^{5}$ $\rightarrow N^{5}=H(2,4) / T$. In order to identify $N^{5}$, consider $R P^{2} \times R P^{4}$ as $R P(5)$, the projective space bundle of a trivial 5-plane bundle over $R P^{2}$, and observe that the defining relation $\Sigma_{0}^{2} x_{i} y_{i}=0$ gives orthogonality, i.e. $H(2,4)=R P\left(\lambda^{\perp}+2\right)$ where $\lambda^{\perp}$ is the orthogonal complement of the line bundle $\lambda$ inside 3. $T$ is multiplication by -1 in the fibers of 2 , and so $N^{5}$ is the quotient of $S\left(\lambda^{\perp}+2\right)$ by the $Z_{2} \times Z_{2}$ given by -1 in the fibers of $\lambda^{\perp}$ and by -1 in the fibers of 2 , the product of these being -1 on the sphere. Thinking of $S\left(\lambda^{\perp}+2\right)$ as the fiberwise join $S\left(\lambda^{\perp}\right) * S(2), S\left(\lambda^{\perp}+2\right) / Z_{2}$ $\times Z_{2}$ is $S\left(\mu_{2} \lambda^{\perp}\right) * S\left(\mu_{2} 2\right)=S\left(\mu_{2} \lambda^{\perp}+\mu_{2} 2\right)=S\left(\mu_{2} \lambda^{\perp}+2\right)$. Now $\mu_{2} \lambda^{\perp}+2 \cong \lambda+$ 3, being 4-plane bundles with the same Stiefel-Whitney class and so $N^{3}$ is the normal sphere bundle of $R P^{2}$ imbedded in $R^{6}$, hence a framed manifold.

One may generalize this contruction for $H^{5}$. Noting that $4 \lambda \cong 4$ over $R P^{2}$, $H^{5} \cong R P\left(\lambda^{\perp}+2\right)=R P(3 \lambda+1)=R P(\lambda+3)$ and branches over

$$
S\left(\mu_{2}(\lambda+1)+\mu_{2} 2\right) \cong S(\lambda+1+2)=S(\lambda+3) .
$$

Specifically, for $n=2^{k}+2^{q}-1$, one may consider

$$
Q^{n}=R P\left(\left(\lambda_{1}+1\right)+\left(\lambda_{2}+1\right)\right)
$$

over $R P^{2^{k}-2} \times R P^{2^{q}-2}$ as branching over $U^{n}=S\left(\left(\lambda_{1}+1\right)+\left(\lambda_{2}+1\right)\right)$. One notes that $w\left(U^{n}\right)=1$, and that $U^{n}$ is frameable for $3 \geqslant k>q \geqslant 1$. By [17, Lemma 3.4] $Q^{n}$ is indecomposable in $\mathfrak{N}_{*}$ and one may check very painfully that $w_{n+2-2^{q+1}} w_{2}^{2^{q}-1}\left[Q^{n}\right]$ $\neq 0$ to see that $Q^{n}$ is cobordant to $H^{n}$. Thus, one actually has branchings over manifolds having $w=1$ in every exceptional dimension, and over framed manifolds when $n=5,9$, and 11 .

\section{REFERENCES}

1. M. F. Atiyah, Bordism and cobordism, Proc. Cambridge Philos. Soc. 57 (1961), 200-208.

2. I. Berstein and A. L. Edmonds, The degree and branch set of a branched covering, Invent. Math. 45 (1978), 213-220. $1-10$.

3. N. Brand, Necessary conditions for the existence of branched coverings, Invent. Math. 54 (1979),

4. , Classifying spaces for branched coverings, Indiana Univ. Math. J. 29 (1980), 229-248.

5. J. Brillhart, J. Tonascia and P. Weinberger, On the Fermat quotient, Computers in Number Theory (A. O. L. Atkin and B. J. Birch, eds.), Academic Press, New York, 1971, pp. 213-222.

6. P. E. Conner and E. E. Floyd, Differentiable periodic maps, Springer-Verlag, Berlin, 1964.

7. A. L. Edmonds, Orientability of fixed point sets, Proc. Amer. Math. Soc. 82 (1981), 120-124.

8. A. Hattori, Genera of ramified coverings, Math. Ann. 195 (1972), 208-226.

9. F. Hirzebruch, The signature of ramified coverings, Global Analysis, Papers in honor of K. Kodaira, Univ. of Tokyo Press, Tokyo and Princeton Univ. Press, Princeton, N. J., 1969, pp. 252-265. 
10. N. H. Kuiper, The quotient space of $\mathbf{C P}(2)$ by complex conjugation is the 4-sphere, Math. Ann. 208 (1974), 175-177.

11. P. S. Landweber, Fixed point free conjugations on complex manifolds, Ann. of Math. (2) 86 (1967), 491-502.

12. C. N. Lee and A. Wasserman, Equivariant characteristic numbers, Proc. 2nd Conf. Compact Transformation Groups (Univ. of Mass., Amherst, 1971), Lecture Notes in Math., vol. 298, SpringerVerlag, Berlin and New York, 1972, pp. 191-216.

13. M. Nakaoka, Homology of the infinite symmetric group, Ann. of Math. 73 (1961), 229-257.

14. Note on cohomology algebras of symmetric groups, J. Math. Osaka City Univ. 13 (1962), 45-55.

15. H. L. Rosenzweig, Bordism of involutions on manifolds, Illinois J. Math. 16 (1972), 1-10.

16. R. E. Stong, Complex and oriented equivariant bordism, Topology of Manifolds (J. C. Cantrell and

C. H. Edwards, Jr., eds.), Markham, Chicago, Ill., 1970, pp. 291-316.

17. On fibering of cobordism classes, Trans. Amer. Math. Soc. 178 (1973), 431-447.

18. C. T. C. Wall, Determination of the cobordism ring, Ann. of Math. 72 (1960), 292-311.

Department of Mathematics, University of Virginia, Charlottesville, Virginia 22903 